\title{
Dictynna
}

Dictynna

Revue de poétique latine

4 | 2007

Varia

\section{Échos de Sappho et éléments nuptiaux dans les Odes d'Horace}

Olivier Thévenaz

\section{(2) OpenEdition}

Journals

Édition électronique

URL : http://journals.openedition.org/dictynna/155

DOI : 10.4000/dictynna.155

ISSN : 1765-3142

Référence électronique

Olivier Thévenaz, «Échos de Sappho et éléments nuptiaux dans les Odes d'Horace », Dictynna [En ligne], 4 | 2007, mis en ligne le 25 novembre 2010, consulté le 10 septembre 2020. URL : http:// journals.openedition.org/dictynna/155; DOI : https://doi.org/10.4000/dictynna.155

Ce document a été généré automatiquement le 10 septembre 2020.

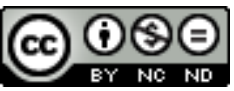

Les contenus des la revue Dictynna sont mis à disposition selon les termes de la Licence Creative Commons Attribution - Pas d'Utilisation Commerciale - Pas de Modification 4.0 International. 


\title{
Échos de Sappho et éléments nuptiaux dans les Odes d'Horace ${ }^{1}$
}

\author{
Olivier Thévenaz
}

\section{Introduction : la réception de Sappho entre poésie érotique et chants de mariage}

1 Quand on travaille sur la réception de Sappho, on est amené à constater qu'elle se structure en deux volets distincts, mais qui entrent régulièrement en dialogue l'un avec l'autre : le groupe particulier des poèmes nuptiaux, précisément définis par l'occasion à laquelle ils se réfèrent, et l'ensemble plus général des poèmes qui se rapportent aux relations érotiques à l'intérieur du chœur de jeunes filles dirigé par Sappho². Si l'on étudie le contexte de ce cercle, cette bipartition, voire opposition est certes artificielle : non seulement ces deux types de poèmes partagent un certain nombre de traits, comme la mise en valeur de la grâce des $\Pi \alpha \rho \theta \varepsilon$ ćvor et l'expression de leur attrait érotique, mais ils doivent aussi être considérés comme compatibles l'un avec l'autre, vu que le groupe de jeunes filles - institution pour laquelle Sappho, elle-même mariée, était reconnue - leur offrait une initiation notamment érotique censée préparer leur réintégration à la communauté en tant qu'épouses. Cette distinction devient toutefois pertinente dès que l'on envisage la réception. Alors que l'occasion des chants de mariage restait évidente, le contexte précis des poèmes homoérotiques de Sappho a dû rapidement devenir insaisissable. Ces deux ensembles ont ainsi pu paraître refléter des formes d'éros antithétiques et avoir une transmission différente, le premier (avec peutêtre d'autres textes à forte dimension pragmatique) grâce à des recueils de poèmes pouvant être repris comme modèles ou tels quels dans des circonstances analogues, le second par l'intermédiaire du banquet, qui donna à ces textes une nouvelle vie ${ }^{3}$. En tout cas, les chants de mariage semblent avoir un statut particulier dans le recueil alexandrin des poèmes de Sappho, étant certainement regroupés à la fin des livres à unité métrique et probablement intitulés Épithalames, voire - pour les inclassables réunis dans un neuvième et dernier volume de poèmes en mètres variés portant ce 
titre ${ }^{4}$. L'édition alexandrine fixe ainsi la distinction entre ce qui est nuptial et ce qui ne l'est pas.

Dans les résonances des poèmes de Sappho que l'on peut percevoir chez différents auteurs surtout à partir de l'époque alexandrine, on retrouve de façon intéressante cette distinction, certains textes ou certains genres évoquant par exemple exclusivement l'un ou l'autre de ces ensembles, alors que des échos des deux groupes sont mis en dialogue dans d'autres œuvres. Théocrite, à l'époque même de la constitution du recueil alexandrin, reprend ainsi dans son Épithalame d'Hélène (Idylle XVIII) plusieurs motifs des chants nuptiaux de Sappho et dans d'autres Idylles des éléments non-nuptiaux (entre autres le fragment 31 et ses effets physiques de l'amour), mais présente aussi des évocations combinées : le meilleur exemple est celui du Cyclope (Idylle XI), où Polyphème, rêvant d'épouser Galatée, tente de chanter son propre chant de mariage, mais prononce en fait une plainte, analogue à celles de Sappho, à propos d'un amour non réciproque 5 . À Rome, c'est surtout Catulle qui met en dialogue des échos des deux volets de la poésie de Sappho: si d'une part ses deux épithalames (61 et 62) se rapprochent notamment par leurs comparaisons de certains fragments de chants de mariage de Sappho, si d'autre part le poème 51 est une quasi-traduction du fragment 31 de Sappho, le seul autre poème de Catulle en strophes sapphiques, le poème 11 décrivant la rupture avec la puella (qui n'est même plus nommée Lesbia), se clôt en détournant une image nuptiale de Sappho ${ }^{6}$.

Ce survol très rapide de quelques moments essentiels de la réception de Sappho donne une idée de la tradition dans laquelle s'inscrivent les échos de ses poèmes chez Horace, auxquels je veux ici m'intéresser plus en détail. En effet, le rôle de Sappho est loin d'être marginal chez Horace, même s'il choisit Alcée comme modèle privilégié parmi les poètes de Lesbos : en relisant avec attention les textes qui illustrent à première vue cette préférence d'Horace pour Alcée, la recherche récente a montré qu'elle n'est pas aussi nette, et que Sappho ne s'efface pas derrière lui ; vu le binôme qu'ils forment, le choix explicite d'Alcée permet même à Horace de choisir aussi implicitement Sappho, dont Catulle avait fait son modèle; et la relation d'Horace à Sappho est d'autant plus intéressante qu'elle le situe aussi par rapport à ce prédécesseur dont il passe sous silence les expérimentations lyriques pour se proclamer, dans l'ode 3.30, le premier à adapter les poètes éoliens en latin ${ }^{7}$. Mais contrairement à Catulle, Horace n'évoque apparemment pas le volet nuptial de l'œuvre de Sappho, et sa lyrique érotique ne fait en général pas référence à des relations matrimoniales. C'est pourtant le thème du mariage que je veux étudier ici, considérant que cet aspect joue malgré tout un rôle dans la définition de la voix lyrique d'Horace par rapport à la tradition et aux poètes contemporains. Avant de me concentrer dans cette optique sur les trois premiers livres des Odes, je partirai de l'Ode 4.1, car sa position à l'ouverture du second recueil en fait pour Horace un moment de réflexion sur sa lyrique, notamment érotique, qu'il reprend après un intervalle de dix ans.

\section{Sappho et Vénus entre amour et mariage à l'ouverture du livre IV des Odes d'Horace}

Avec l'Ode 4.1, Horace ne rattache pas seulement son nouveau recueil à sa lyrique antérieure, mais aussi à la tradition grecque, représentée en l'occurrence par Sappho. En effet, la référence au passé du je lyrique, d'une part, est marquée par une auto- 
citation modifiée de telle façon qu'elle évoque le topos de l'amour doux-amer, d'origine sapphique ${ }^{8}$. D'autre part et surtout, Horace refait au début de ce livre la prière à Aphrodite placée par les éditeurs alexandrins en tête du recueil des poèmes de Sappho, position qui lui donnait une valeur programmatique d'ailleurs étrangère au contexte initial de son énonciation: l'hymne clétique de la poétesse de Lesbos devient ici un hymne apopemptique à Vénus, dans lequel la déesse est priée non de venir à nouveau auprès du je et de combattre à ses côtés, mais de l'épargner dans la guerre qu'elle mène à nouveau contre lui et de partir où l'appellent les prières des jeunes gens; et alors que Sappho reprend sa demande initiale dans la troisième et dernière partie du poème, symétrique à la première, Horace y introduit malgré tout un nouveau cycle érotique, marqué par un écho des symptômes de la passion amoureuse décrits par Sappho dans le fragment $31^{9}$. Horace entame ainsi ce recueil par une sorte de recusatio paradoxale des aspects érotiques pourtant constitutifs de sa propre lyrique: il s'agit même d'un détournement de la recusatio, réservée d'ordinaire au refus des genres élevés; mais tout comme ce stratagème permet souvent à Horace de célébrer les puissants tout en disant qu'il ne le fait pas - il y en a plusieurs exemples dans le premier recueil, et encore un juste après dans l'ode 4.2 -, il sert ici de prélude à de nouveaux poèmes lyriques notamment érotiques ${ }^{10}$. Or, cette anti-recusatio lui donne en même temps l'occasion de faire l'éloge de Paulus Maximus, futur membre de la famille impériale par son prochain mariage avec Marcia, petite-nièce de Jules César et cousine d'Auguste ; et s'il est vrai qu'Horace se réfère ici à ce mariage, on a bien, dans un contexte marqué par la présence de Sappho, un contraste entre la passion érotique s'emparant du poète qui n'en a plus l'âge - un amour bisexuel, asymétrique et éphémère typique des banquets grecs - et l'amour matrimonial romain du noble jeune homme vers lequel le je lyrique cherche à détourner Vénus - une relation décrite en des termes qui en font d'ailleurs une sorte d'idéal élégiaque ${ }^{11}$.

Avant de me pencher sur les échos de Sappho et les éléments nuptiaux du premier recueil lyrique d'Horace, je voudrais souligner un second point sur lequel le livre IV est plus explicite que le premier recueil, dont il révèle pour ainsi dire un aspect latent. Il s'agit du rôle de Vénus et du thème érotico-matrimonial. La recusatio inversée du poème d'ouverture introduit tant des poèmes de célébration, prédominants dans la première moitié du livre (4.2, 4.4 et 4.5 , ainsi que $4.6,4.14$ et 4.15$)$, qu'un cycle érotique (ou plus généralement de poésie légère), qui sera surtout présent dans la seconde (4.104.13 , et déjà 4.7 ) ; en outre, ce poème est le premier d'un groupe d'odesqu'on pourrait dire " poétologiques » en ce qu'elles articulent assez explicitement une réflexion sur la poésie (4.1-4.3, 4.6, 4.8, 4.9 et 4.15). Ces différents arcs se rejoignent à l'autre extrémité du livre dans l'ode 4.15, l'épilogue adressé à Auguste, qui commence comme une recusatio de l'épopée au profit de la poésie humble justifiée par l'intervention d'Apollon, continue par un éloge des actes pacificateurs du princeps et se termine dans l'otium qui permet au poète de chanter sur un mode lyrique, dans la joie de Bacchus et celle de la famille traditionnelle, la Vénus des origines de Rome et donc aussi, malgré tout, de la poésie sublime latine. Le rôle de Vénus est de faire le lien entre ces thématiques. La déesse a dans la tradition lyrique une fonction centrale, principalement non-nuptiale, en lien avec le volet érotique de cette poésie, dont l'emblème est Sappho; mais elle veille aussi sur la gens Iulia qui descend d'elle, et au-delà de cette dernière sur la postérité de Rome dont Auguste a récemment assuré la stabilité par ses lois sur les mariages et sur les adultères. Horace ne choisit pas entre les deux: il peut certes paraître paradoxal qu'il présente comme idéal un amour de type matrimonial et 
pratique lui-même une lyrique érotique traditionnellement non-nuptiale, mais les deux participent en fait de l'éloge d'Auguste, qui a rétabli l'otium nécessaire à une telle poésie ; et nous verrons que le mariage est non seulement un idéal moral, mais aussi un moment naturel et rituel vers lequel tend métaphoriquement la lyrique d'Horace, même érotique et symposiaque ${ }^{12}$. J'illustrerai ces différents points dans les trois premiers livres des Odes, en commençant par les aspects moraux, certes moins explicites vu qu'ils précèdent les lois morales de 18 et 17 av. J.-C.

\section{Le mariage comme idéal moral}

6 C'est la dernière des odes romaines, l'Ode 3.6, qui chez Horace idéalise le plus clairement le lien sacré du mariage: après quatre premières strophes évoquant les maux qui continueront de s'abattre sur Rome tant qu'elle n'aura pas changé de comportement religieux et moral, et avant quatre autres présentant le déclin moral de Rome, de génération en génération, depuis les guerres Puniques, les quatre strophes centrales sont occupées par la description de l'actuelle souillure des mariages et de la société, illustrée par le comportement immoral d'une vierge à peine mûre pour le mariage, qui plus tard commet l'adultère au vu et au su de son mari buveur, se faisant payer son déshonneur ${ }^{13}$. Cet exemple est intéressant: le contexte évoqué - avec les danses d'Ionie et les artifices auxquels prend plaisir la jeune fille, avec l'identification de son âge par l'adjectif matura malgré son "ongle tendre", puis avec les amours adultères et le cadre du banquet - rappelle en effet celui des poèmes érotiques d'Horace, à la différence que cette femme est de condition libre, mariée, et qu'elle se comporte avec autant, voire plus de licence qu'une courtisane. Horace célèbre donc ici l'idéal moral visé par les réformes d'Auguste, tout en soulignant, entre la décadence romaine qu'il décrit et les amours de sa lyrique, des parallèles en termes de licence artistique et morale, mais aussi une différence claire, à savoir que sa poésie d'amour ne met pas en scène des femmes libres et mariées. L'institution romaine du mariage constitue ainsi à la fois un idéal moral et une ligne de démarcation par rapport aux amours de la poésie lyrique, d'inspiration grecque et symposiaque.

On trouve au livre III, dans le cycle des odes romaines et en-dehors, d'autres allusions politiques au mariage, parmi lesquelles une, dans l'ode 3.3, me paraît mériter d'être soulignée davantage ${ }^{14}$. Après quatre strophes célébrant la justice et la fermeté qui valent son apothéose à Auguste, comme avant lui à Romulus-Quirinus, Horace rapporte le discours par lequel Junon admet ce dernier parmi les dieux et renonce à sa haine contre les Troyens devenus Romains ; mais elle commence en rappelant que la chute d'Ilion est due surtout à l'adultère de Pâris et Hélène, et finit en assortissant son pardon de la condition que Rome ne relève pas Troie, ce symbole de dépravation et d'orgueil, faute de quoi elle, «épouse et sœur de Jupiter ", la détruirait à nouveau ${ }^{15}$. En se posant ainsi en garante des mariages et de la stabilité sociale, Junon s'oppose certes à Vénus, dont le pouvoir délétère est représenté ici par le jugement de Pâris et par l'adultère dans lequel il entraîne Hélène, mais elle s'associe aussi à sa descendance, en acceptant Romulus et en soutenant la politique d'Auguste.

8 Si c'est surtout au livre III, et dans les odes romaines en particulier, que le mariage apparaît comme un idéal moral, cet aspect n'est toutefois pas absent des deux premiers livres. Il est même introduit comme tel dès l'Ode 1.15 avec le même exemple de Pâris et Hélène. Ce poème consiste en un récit dont l'essentiel est occupé par la prophétie que 
Nérée adresse à Pâris tandis qu'il enlève Hélène : il lui annonce que la Grèce viendra rompre ses "noces" contractées sous de mauvais auspices et qu'il ne pourra plus compter sur l'abri de sa "chambre nuptiale", lexique matrimonial qui fait d'autant plus ressortir le vers central condamnant comme adultère le protégé de Vénus ${ }^{16}$. La présence du thème du mariage dans ce qui n'est pas un poème érotique, mais une ode narrative à sujet épique, permet d'établir une définition morale du mariage comme un lien dont la rupture ou l'usurpation ont des conséquences néfastes. Ainsi, ce poème forme avec l'ode 3.3 un diptyque lié au mariage et à l'exemple épique de Pâris et Hélène, dont les conclusions morales seront reprises par l'ode 3.6.

Mais comme l'ode 3.6 - je l'ai signalé plus haut - présente des résonances détournées des poèmes d'amour d'Horace, l'Ode 1.15 tisse aussi des liens avec le volet érotique de sa lyrique. Il se trouve d'abord que Pâris, l'anti-héros épique, apparaît aussi comme un imposteur poétique: il égrène "sur une lyre pacifique des chansons agréables aux femmes » dans sa chambre nuptiale, mais s'avèrera incapable de tenir ses promesses à Hélène en fuyant mollement face à Diomède comme un cerf qui oublie son herbage à la vue d'un loup; cette comparaison, que l'on peut qualifier d'anti-nuptiale et qui contraste avec l'évocation de la lyrique érotique de Pâris, suggère que le mariage a peut-être une signification métaphorique pour la poésie ${ }^{17}$. Ensuite, la figure d'Hélène relie cette ode aux deux suivantes, d'inspiration érotique, qui sont des invitations très différentes à la réunion des amants par le chant : l'ode 1.16, inspirée probablement de la palinodie à Hélène de Stésichore, montre le je rétracter ses iambes injurieux pour tenter de regagner le cœur de la fille à laquelle il s'adresse; quant à l'ode 1.17, elle invite Tyndaris, dont le nom rappelle Hélène fille de Tyndare, à quitter son amant violent pour venir auprès du poète chanter sur la lyre d'Anacréon « les tourments, pour le même homme, de Pénélope et de la cristalline Circé $»^{18}$. Enfin, la dialectique mariage/ adultère, présente en lien avec la lyre au début et à la fin de ce groupe - à propos des amours de Pâris et d'Ulysse - est peut-être déjà annoncée à la fin de l'ode 1.13, qui formule un idéal d'union sans rupture jusqu'à la mort sur lequel je reviendrai plus loin $^{19}$. Reste cependant à définir le rapport exact entre cette isotopie nuptiale et la lyrique érotique d'Horace, essentiellement non-matrimoniale.

À ce propos, il convient de mentionner encore un poème du livre II qui, au centre presque parfait du recueil, associe thème érotique et célébration politique dans un programme poétique où le mariage a sa place : s'adressant à Mécène pour justifier par la volonté de la Muse son refus des genres élevés, Horace chante dans l'ode 2.12 les " sentiments réciproques et fidèles » de celle qu'il appelle, avec une ambiguïté calculée, sa "maîtresse Licymnia", dont on croit d'abord qu'elle est son amante, mais qui s'avère ensuite être l'épouse de son patron Mécène ; en célébrant en Licymnia-Terentia l'aimée idéale, dont les charmes seraient aussi adaptés à ses propres conventions lyriques qu'elles ne le sont à celles de Mécène, présentées comme élégiaques, Horace fait de la relation matrimoniale d'un couple haut-placé de l'Empire un modèle érotique ${ }^{20}$. Mais on notera bien que le je lyrique n'entretient avec sa domina qu'une relation d'ordre poétique (cantus, dicere), et que l'amour mutuel dont il est question est celui qu'elle entretient avec son époux: on verra d'ailleurs plus loin que ce n'est certainement pas un hasard si le mariage représente la réalisation érotique d'une passion élégiaque, alors qu'il n'est, d'un point de vue lyrique, qu'un idéal poétique métaphorique. 
11 L'Ode 2.12 est précédée dans le livre II par trois odes érotiques évoquant le mariage, dont deux le présentent avec ironie comme un idéal. Tout d'abord, le je de l'ode 2.4, appelant son destinataire Xanthias à ne pas rougir de son amour pour sa servante, lui suggère à moitié sérieusement que la belle, fidèle et vertueuse Phyllis, comme les captives de l'épopée aimées de leurs maîtres, fait peut-être de lui le gendre de nobles beaux-parents ; de plus, il termine en déclarant - mais on peine à le croire - qu'il loue les attraits de la jeune femme en homme intègre, comme le rhéteur Ménandre conseille de le préciser quand on doit prononcer un éloge nuptial ${ }^{21}$. Quant à l'ode 2.8, qui détourne des formules $\mathrm{du}$ poème 61 de Catulle, elle constitue une parodie d'épithalame : la beauté et les serments trompeurs de Bariné, à qui elle est adressée, la font apparaître non comme la « nouvelle épouse ", mais comme le «tourment universel des jeunes hommes "; et la strophe finale du poème la blâme en reprenant les termes par lesquels Catulle fait l'éloge du dieu du mariage Hymen ${ }^{22}$. Ces odes, l'une en positif et l'autre en négatif, mais toutes deux avec ironie, présentent le mariage comme un idéal érotique, qui semble cependant difficile à réaliser, et en tout cas inaccessible au je lyrique. En effet, que ce dernier soit séduit par les attraits de Phyllis ou victime des parjures de Bariné, tenté par l'adultère poétique avec Licymnia ou exposé à d'autres manifestations de l'amour encore, les relations décrites dans les odes érotiques d'Horace sont toutes non-matrimoniales. Mais nous allons voir maintenant qu'elles sont parfois évoquées par des images à connotation nuptiale dont il s'agit de définir la fonction.

\section{Images nuptiales et temporalité de l'amour}

12 L'exemple le plus clair où des connotations nuptiales viennent s'attacher à un amour qui n'a rien de matrimonial se trouve toujours au livre II, et fournit à mon sens une première clé pour interpréter la présence du thème nuptial, indirectement liée à celle de Sappho, dans les odes érotiques d'Horace. Dans l'ode 2.5, où l'absence de destinataire suggère que le poète s'adresse à lui-même, la jeune fille aimée est d'abord présentée comme encore inapte au joug d'une union égale, et assimilée à une génisse qui préfère jouer avec les veaux plutôt que de s'unir au taureau ; cette métaphore nuptiale filée est ensuite prolongée par une autre image - non plus animale, mais végétale - suggérant le mariage, celle du raisin encore vert qui va prendre les couleurs pourpres de l'automne et arriver à maturité ${ }^{3}$. On comprend que le mariage est lié par ces métaphores naturelles au thème du passage du temps, dont les aspects linéaires sont évoqués par la succession des âges de la vie plus ou moins propices à l'amour, et les aspects cycliques par le retour rituel des saisons.

13 À ce point central du poème, la réciprocité érotique est proche, mais on la voit s'éloigner à nouveau dès la strophe suivante, introduite par une phrase qui a fonction de pivot: iam te sequetur semble dans un premier temps signifier que la jeune fille est sur le point de s'attacher à lui ; mais quand vient l'explication de la course du temps, qui donne à la fille les années qu'il enlève à l'homme, on comprend qu'elle va prendre le même chemin que lui, restant derrière lui dans le temps, et qu'il ne sera pas le « mari » que va chercher la jeune fille appelée ici Lalagé ${ }^{24}$. Or, la strophe entière et cette phrase initiale en particulier rappellent les mots d'Aphrodite à Sappho, que celle-ci rapporte dans sa célèbre prière à la déesse : « car si elle fuit, bientôt elle poursuivra... ", lui avait-elle dit naguère pour la consoler de la résistance d'une jeune fille ${ }^{25}$. Et alors 
que la critique moderne hésite sur le sens de cette phrase, qui ne dit pas non plus si l'aimée doit répondre à l'amour de Sappho ou aimer quelqu'un d'autre, Horace paraît pencher pour la seconde interprétation: les adverbes de temps qui scandent l'ode 2.5 (" pas encore », « maintenant », « bientôt ») insistent sur la linéarité de l'amour, déjà suggérée par les deux «bientôt» d'Aphrodite chez Sappho, alors que la poétesse insistait quant à elle davantage, en répétant trois fois "de nouveau » au début de sa prière, sur le temps cyclique de la poésie lyrique, définie par le retour rituel des occasions de chant ${ }^{26}$. Horace montre ainsi que ces temporalités ne coïncident pas et qu'une réciprocité érotique durable est donc impossible pour le je lyrique; et il est frappant de voir qu'il se réfère pour cela au mariage, moment unique dans une vie amoureuse en même temps que circonstance lyrique récurrente, qui se trouvait marquer pour Sappho la séparation définitive d'avec les jeunes filles de son groupe.

On trouve déjà dans plusieurs odes érotiques du livre I de telles images à caractère nuptial à propos de relations non-matrimoniales. Ce paradoxe apparaît peut-être le plus clairement dans l'ode 1.33, qui, à propos de passions non réciproques, détourne l'image matrimoniale du joug, que le jeu cruel de Vénus impose ici à des amants «mal appariés $\aleph^{27}$. Des comparaisons tirées du monde naturel, qui rappellent celles récurrentes dans la tradition des chants de mariage, apparaissent aussi à plusieurs reprises. Ainsi, la Chloé de l'ode 1.23, pareille à un faon apeuré, évite le je lyrique qui ne cherche pourtant pas à la briser comme un lion ou un tigre : cette double comparaison animale commence certes par un écho d'un poème symposiaque d'Anacréon, mais elle est référée au thème, récurrent dans les chants nuptiaux de Sappho et de Catulle notamment, de l'attachement à sa mère de la jeune fille pourtant "mûre pour l'homme » - on trouve déjà ici l'idée des saisons de l'amour mise en évidence dans l'ode 2.5 -, et se rapproche d'images nuptiales analogues ${ }^{28}$. Et à la fin de l'Ode 1.36, Damalis est avec son nouvel amant "plus enveloppante que le lierre lascif », une comparaison hyperbolique végétale rappelant doublement celles récurrentes dans les chants de mariage de Sappho ou de Catulle, mais évoquant ici une relation explicitement adultère ${ }^{29}$.

Parmi les poèmes érotiques du livre III qui évoquent aussi l'idée du mariage à propos de relations non-matrimoniales ou de la temporalité de l'amour, je ne mentionnerai ici que le plus clair exemple, celui de l'ode 3.11. Lydé y est en effet évoquée par une comparaison animale, comme Lalagé dans l'Ode 2.5 ou Chloé dans l'Ode 1.23, en des termes qui précisent bien son caractère nuptial, et ceci bien que cette image fasse aussi clairement écho à un poème érotique symposiaque d'Anacréon: «telle une cavale de trois ans dans les vastes plaines, elle bondit et s'ébat et craint d'être touchée, étrangère à l'hymen et peu mûre encore pour l'audace d'un époux $»^{30}$. L'application à une relation non-matrimoniale d'une image issue certes d'un poème non-nuptial, mais explicitement caractérisée comme nuptiale par la mention de l'hymen et d'un époux, exprime ici une fois de plus la dimension rituelle de la succession des âges de l'amour. Mais Michèle Lowrie a bien montré que le thème du mariage avait ici une fonction métaphorique plus large engageant le rapport de la lyrique d'Horace à ce qu'il définit par contraste comme élégiaque ${ }^{31}$. Je vais maintenant m'engager sur cette piste, en élargissant la réflexion à d'autres odes d'Horace où apparaît l'idée de l'amour éternel. 


\section{Éternité de l'amour ou de la poésie ? La lyrique face à l'élégie}

16 entière faite de codas enchâssées: plus de la moitié du poème relate l'histoire des Danaïdes, dont plus de la moitié est elle-même occupée par le discours final d'Hypermestre ${ }^{32}$. De plus, l'ode est adressée non pas directement à Lydé, mais - en écho à un fragment de Sappho - à la lyre censée la persuader d'écouter ce récit et ce discours en particulier; la jeune fille est ainsi non seulement l'objet de l'amour du je lyrique, mais aussi une figure du public d'Horace, voire de sa poésie elle-même ${ }^{33}$. Par ailleurs, Michèle Lowrie note entre le récit et le cadre une disparité relative au thème du mariage. Certes, à l'inverse des quarante-neuf Danaïdes qui tuent leurs maris pendant leur nuit de noces, la seule à épargner le sien, « digne du flambeau nuptial » et « illustre à jamais ", est un exemple pour Lydés ${ }^{34}$. Mais si cette dernière est invitée à accepter le mariage, Hypermestre interrompt le sien pour permettre à son mari de partir et de survivre, et prend congé de lui en lui demandant de graver sur son tombeau une plainte élégiaque qui s'oppose à la poésie lyriqueproposée au début à Lydé par le je; selon l'explication métapoétique de Michèle Lowrie, ce poème oppose l'élégie, représentée par Hypermestre, qui compense l'interruption de son mariage par une inscription écrite perpétuant son souvenir, à la lyrique qui, entre le je et Lydé, établit un contrat de permanence du chant oral au-delà même de la mort, évoquée par la catabase de la lyre d'Orphée ; le mariage, s'il désigne du côté de l'élégie au sens propre l'union érotique (même éphémère) entre Hypermestre et Lyncée, représente du côté de la lyrique, de façon métaphorique, l'union poétique éternelle que le je propose à Lydé35.

Une telle opposition entre lyrique et élégie peut certes paraître schématique et artificielle, d'autant plus que l'élégie érotique latine - pour ne prendre en compte que le type d'élégie contemporain par rapport auquel Horace prend position - ne présente de loin pas un visage monolithique et n'est pas même définie comme un genre avant Ovide ; mais Michèle Lowrie précise bien que ce n'est pas ici ce courant lui-même qui est en question, mais l'image simplifiée, caricaturale, qu'Horace construit de l'élégie pour en distinguer sa lyrique, en particulier érotique, qui partage par ailleurs bien des traits avec elle ${ }^{36}$. J'aimerais montrer ici qu'Horace souligne la proximité et la distance entre ces types de poésie d'amour, qui évoquent tous deux des relations en principe non-matrimoniales, justement par son traitement du thème du mariage, avec les idées d'unicité et de durabilité qui lui sont liées : alors qu'il est un idéal érotique pour l'élégie, qui transforme une relation éphémère de type symposiaque en une passion unique qu'elle cherche à faire durer (et qui est sa condition d'existence), la lyrique vise moins l'éternité de l'amour que celle de la poésie, dont le mariage est cette fois une métaphore. Comme exemple de cette confrontation entre la lyrique et l'élégie autour $\mathrm{du}$ thème du mariage, j'ai déjà évoqué plus haut l'ode 2.12, où le je lyriquesuggère son union avec Licymnia dans le chant avant de présenter son mariage avec Mécène comme la réalisation d'un idéal érotique élégiaque. J'y ajouterai ici les Odes 1.13 et 1.22, puis 3.9, qui impliquent en outre Catulle, précurseur de l'élégie à Rome et prédécesseur lyrique d'Horace, ainsi que Sappho.

La critique a noté de longue date que l'ode 1.13 était une variation sur le thème des manifestations de la passion amoureuse décrites dans le fragment 31 de Sappho et reprises par Catulle dans son poème $51:$ le discours d'Horace se rapproche de celui de 
Sappho non seulement par les symptômes physiques qu'éprouve le je, mais peut-être surtout par le regard presque extérieur qu'il porte sur eux ${ }^{37}$. Il faut cependant préciser que la nature de ces symptômes est certainement différente : ceux décrits par Horace sont des signes clairs de jalousie, alors que ceux de Sappho, difficiles à identifier vu notre ignorance du contexte de ce poème, expriment probablement les sensations que provoque en elle l'amour pour la jeune fille plutôt que la jalousie de l'homme assis en face de cette dernière ; en tout cas, Horace prend une posture plus marquée que Catulle dans sa quasi-traduction, débutant non par une célébration du bonheur du « rival » aux

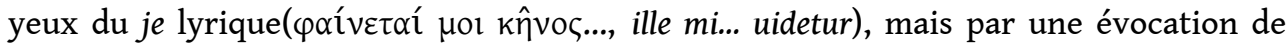
l'attitude du tu envers Télèphe (cum tu... Telephi), qui provoque une interjection de dépit du je (uae) ${ }^{38}$. Enfin, l'enjeu de ce poème d'Horace est sans doute autant - si ce n'est plus - son rapport à Catulle que le lien avec Sappho: j'en veux pour preuve le fait qu'il résume les premiers symptômes traduits par Catulle en un mot (mens), qu'il poursuit sa liste là où Catulle interrompait sa traduction, en reprenant avec le teint pâle et la sueur la quatrième strophe du poème de Sappho, et qu'il rajoute encore d'autres éléments topiques déjà présents dans d'autres poèmes de Sappho, comme le feu de la passion et l'amour doux-amer ${ }^{39}$.

19 Mais de même que Sappho et Catulle - différemment - changeaient de voix pour finir leur poème, Horace adopte à la quatrième strophe un autre ton, qu'il modifiera encore à la dernière. Si les trois premières strophes présentaient un discours dans le style de l'épigramme érotique hellénistique, la quatrième est une mise en garde contre l'espoir qu'une passion douce-amère comme celle de Lydia et Télèphe dure toute la vie: il dénonce ainsi comme illusoire l'idéal érotique catullien d'aeternum sanctae foedus amicitiae, cet idéal quasi matrimonial qui sera repris par les élégiaques ${ }^{40}$. Enfin, ce ton de praeceptor amoris anti-élégiaque laisse la place dans la dernière strophe à une célébration tout en distance du bonheur idéal que représente une union sans rupture ni plaintes élégiaques : on retrouve le $\mu \alpha \kappa \alpha \rho \imath$ pó $\varsigma$ qui manquait au début du poème, et sa formulation rappelle les vœux des chants de mariage, «Heureux trois fois et davantage... $»^{41}$. En un seul poème, on passe ainsi d'une expression de jalousie exacerbée à des conseils de prudence face à l'espoir qu'un amour passionné dure éternellement, pour finir sur la bénédiction d'une union idéale. Or, Catulle n'est peutêtre pas étranger à ce mouvement: si son idéal est de faire d'un amour non-nuptial passionné un foedus amicitiae aussi durable et sacré qu'un mariage, sa relation avec Lesbie connaît en réalité la rupture, soulignée par l'inversion d'une image nuptiale de Sappho à la fin du poème 11; dans l'ode 1.13, Horace part d'une jalousie rappelant celle qui, dans le poème 51 de Catulle, inaugure le «roman » de Lesbie, mais en retourne ensuite en quelque sorte le cours pour passer non pas avec amertume de l'espoir d'un amour éternel à la séparation, mais avec détachement de la conscience que la passion est éphémère à la célébration, si paradoxale soit-elle, d'une union sans rupture ni plaintes.

On s'est souvent demandé si la fin du poème d'Horace devait ou non être prise au sérieux ${ }^{42}$. À cette question essentielle et récurrente à propos de divers aspects de l'œuvre d'Horace, je crois qu'il n'y a pas de réponse tranchée : il faut penser qu'ironie et sérieux ne s'excluent pas, et cohabitent même souvent chez lui. Dans le cas présent, l'idéal d'un amour réciproque et durable analogue au mariage est certainement inatteignable, ne serait-ce que si on le contemple à la lumière de la réalité érotique décrite dans les quatre premières strophes du poème en termes de passion, de jalousie, de douceur et de cruauté ; en ce sens, la dernière strophe est ironique. Mais même s'il 
est inatteignable, cet idéal peut en rester un: tout en faisant de la rupture et des plaintes les preuves que l'espoir élégiaque de faire durer éternellement un amour nonmatrimonial est une illusion, Horace annonce ici, au début de son recueil lyrique, qu'un amour plus serein est mieux à même d'atteindre une forme de stabilité. Sa lyrique érotique, ancrée dans le contexte du banquet aux amours multiples et passionnées, tend vers cet idéal comme vers une asymptote; elle ne pourra l'atteindre que de façon métaphorique et virtuelle, dans l'éternité non de l'amour, mais de la poésie qui chante l'amour.

21 On a vu que l'ode 1.13 faisait écho non seulement au fragment 31 de Sappho, mais aussi à sa quasi-traduction par Catulle dans le poème 51, et qu'elle finissait en un mouvement inverse à celui du pendant de ce dernier, le poème 11. Or, la réponse d'Horace aux poèmes sapphiques de Catulle a un second volet. En effet, l'ode 1.22 se termine par une reprise du poème 51 de Catulle et de son modèle sapphique, mais peut être lue dans son ensemble comme une réécriture du poème 11 ; pour être plus précis, le lien entre ces deux poèmes de six strophes sapphiquesest établi au début par le thème du danger des lieux hostiles par rapport à une relation érotique et par une anaphore de siue $e^{43}$. Mais les différences sont décisives : si Catulle envoie Furius et Aurélius, qui l'accompagneraient dans les expéditions les plus extrêmes, congédier sa puella, Horace se présente au contraire comme à l'abri tant qu'il chante Lalagé ; et alors que le poème du Véronais a une structure asymétrique qui reflète la rupture, faisant se succéder quatre strophes d'adresses anaphoriques, une de malédiction et une de réflexion mélancolique, celui du poète de Venouse est au contraire parfaitement balancé, composé de trois parties égales et symétriques, dont la première énonce une maxime, que la seconde illustre, avant que la troisième, reprenant l'anaphore de la première, donne une conclusion inattendue, d'ordre érotico-poétique plutôt que moral.

C'est la conclusion des deux poèmes qui m'intéresse plus spécialement ici, car le rapport à Sappho s'y cristallise. J'ai déjà dit que Catulle transformait dans sa strophe finale une comparaison nuptiale de Sappho en une image de rupture. Pour y répondre, Horace remplace cet écho par un autre, qui renvoie au fragment 31, corrigeant au passage la version que Catulle lui-même en avait donnée : les deux derniers vers de l'ode rajoutent au dulce ridentem du poème 51 de Catulle la clausule dulce loquentem pour traduire les deux participes de Sappho («parlant doucement et souriant avec charme »), dont son prédécesseur n'avait rendu que le second ${ }^{44}$. Ainsi, alors que dans l' Ode 1.13, il terminait sa reprise non-nuptiale du poème 51 de Catulle en célébrant un idéal matrimonial, Horace récrit ici le poème 11 en substituant à l'image nuptiale de Sappho inversée par Catulle un élément non-nuptial issu du fragment 31 de Sappho et du poème 51 de Catulle. Or, ce mouvement inverse conduit ici aussi à l'expression d'un idéal d'amour durable : là où le poème 11 de Catulle marquait par l'inversion d'une image nuptiale de Sappho la rupture définitive d'avec la puella, Horace dit qu'il aimera partout et toujours Lalagé, en qui se réalise l'idéal inatteignable et virtuel célébré à la fin de l'ode 1.13. Mais cette Lalagé diffère de la puella de Catulle ou des élégiaques en ce qu'elle est objet de chant (v. 10 : dum meam canto Lalagen), et de chant détaché (v. 11 : curis... expeditis), avant d'être objet d'amour ; et quand il est question à la fin de l'amour qu'elle inspire au je lyrique (v. 23-24: dulce ridentem Lalagen amabo, / dulce loquentem), l'explicitation du nom de Lalagé "parlant doucement» en fait une incarnation de la poésie d'Horace. 
De ces deux réponses d'Horace à Catulle, qui font appel à l'autorité de Sappho, la première dénonce l'illusion élégiaque de faire durer éternellement une passion nonnuptiale et y oppose la célébration d'une union de type matrimonial, idéal érotique pourtant inatteignable au poète lyrique, alors que la seconde montre que cet idéal peut se réaliser dans une Lalagé, c'est-à-dire dans un amour qui est avant tout un chant. Il me semble qu'une autre ode dans laquelle l'union éternelle apparaît de façon paradoxale comme un idéal peut aussi être interprétée de cette manière, et liée autant à Sappho qu'au modèle nuptial. Il s'agit de l'ode 3.9 :

'Donec gratus eram tibi

nec quisquam potior bracchia candidae ceruici iuuenis dabat,

Persarum uigui rege beatior.'

'Donec non alia magis

arsisti neque erat Lydia post Chloen, multi Lydia nominis,

Romana uigui clarior Ilia.'

'Me nunc Thressa Chloe regit,

dulcis docta modos et citharae sciens, 10

pro qua non metuam mori,

si parcent animae fata superstiti.'

'Me torret face mutua

Thurini Calais filius Ornyti, pro quo bis patiar mori,

si parcent puero fata superstiti.'

'Quid si prisca redit Venus

diductosque iugo cogit aeneo, si flaua excutitur Chloe

reiectaeque patet ianua Lydiae?'

'Quamquam sidere pulchrior

ille est, tu leuior cortice et inprobo

iracundior Hadria,

tecum uiuere amem, tecum obeam lubens.'

L'Ode 3.9 est l'un des rares poèmes mimétiques du recueil lyrique d'Horace et le seul exemple de chant amébée chez lui : un je anonyme identifiable avec le poète assume les strophes impaires, une femme du nom de Lydia les strophes paires, qui reprennent les principaux traits de syntaxe et de style utilisés dans la strophe précédente en surenchérissant, sauf pour la sixième et dernière strophe, qui répond à la question que pose la cinquième. De ces trois paires de strophes, la première décrit un passé d'union idéalisée précédant l'intervention de rivaux, la deuxième un présent de soumission érotique totale du poète à Chloé et de Lydia à Calaïs, tandis que la troisième évoque, par la question du poète, l'hypothèse d'un retour à "la Vénus d'autrefois » et au "joug d'airain ", ce que Lydie envisage envers et contre tout comme un idéal : le poème se clôt ainsi comme il s'était ouvert, la réponse finale de l'amante, tecum uiuere amem, tecum obeam lubens, faisant écho aux mots initiaux de l'amant, donec gratus eram tibi. Malgré son apparente simplicité, il est virtuellement impossible de saisir la voix poétique qui s'exprime dans ce jeu de rôles plein de références à d'autres textes et discours. C'est ce que montre Jürgen Paul Schwindt dans l'article qu'il consacre à cette ode, et qui passe en revue notamment les analogies avec le poème 45 de Catulle, problématiques et mises en évidence de longue date, ainsi que le rapprochement nouveau qu'il propose avec le dialogue du fragment 137 de Sappho; sans vouloir remettre en question la pertinence 
de ces différents points de comparaison possibles, j'aimerais pour ma part insister sur le modèle nuptial ${ }^{45}$.

On aura en effet noté que l'idée d'union pour la vie et jusqu'à la mort exprimée dans le dernier vers rappelle l'idéal nuptial de la fin de l'ode 1.13, et qu'elle est annoncée dans la strophe précédente par l'image matrimoniale du joug (déjà observée dans les Odes 1.33 et 2.5 notamment), qui renvoie à la « Vénus d'autrefois ». Certes, si l'on revient à cet ancien lien et à la première paire de strophes, on ne note à première vue rien de vraiment nuptial: lié à un amour encore unique et sans rival, l'adjectif initial gratus peut évoquer aussi bien la xópıı des jeunes gens nubiles chez Sappho qu'un désir nonmatrimonial. Mais ces deux premières strophes du poème sont liées à la dernière par un trait de style récurrent, la comparaison hyperbolique : on en trouve une référée au locuteur à la fin des deux premières répliques ("plus fortuné que le roi des Perses", "plus illustre que la Romaine Ilia»), et une série de trois dans la réponse finale de Lydia, dont une positive décrivant Calaïs et deux négatives qualifiant son interlocuteur («Bien que lui soit plus beau qu'un astre, toi plus léger que le liège et plus irritable que l'Adriatique sans frein... »). Or, les comparaisons hyperboliques, en particulier en liste, sont un trait d'éloge typique des poèmes nuptiaux de Sappho, comme le laisse entendre le traité Sur le style du pseudo-Démétrios, qui en cite deux, d'autres étant connues grâce à un rhéteur byzantin ${ }^{46}$. Ce tableau se complique et s'enrichit au vu d'un intermédiaire bucolique : les hyperboles nuptiales de Sappho sont en effet reprises par le Cyclope de Théocrite dans son effort pathétique et grotesque pour se faire accepter comme époux par Galatée, et récupérées de là par Virgile, qui introduit l'opposition d'une série négative d'hyperboles à une positive pour composer un chant amébée, un intertexte à mon sens pertinent pour le poème d'Horace ${ }^{47}$. Or, la forme amébée est aussi employée par Catulle dans un poème mêlant éléments bucoliques et échos nuptiaux de Sappho, le poème 62, qui constitue un autre point de comparaison intéressant: il s'agit de son second épithalame, dans lequel un chœur de jeunes filles et un autre de jeunes gens se répondent à propos du mariage. Cette tradition bucolico-épithalamique n'est ainsi peut-être pas étrangère au fait qu'Horace emploie dans ce poème des comparaisons hyperboliques et une forme amébée.

Il reste cependant un paradoxe à éclaircir à propos des hyperboles nuptiales de la dernière strophe : pourquoi Lydia désire-t-elle vivre et mourir avec le poète, objet d'un anti-éloge, et non avec Calaïs, pourtant plus beau qu'un astre? On peut trouver une partie de réponse dans le couple de strophes médian, qui décrit la situation présente à travers laquelle est perçu tant le passé que le futur. Il n'y apparaît pas de telles comparaisons, mais d'autres éléments suggérant la présence de Sappho : je pense d'une part aux qualités musicales de Chloé, dignes d'une puella docta néotérique et élégiaque connaissant les poèmes de Sappho, et d'autre part - forme différente d'hyperbole - au motif de la mort amoureuse, qui trouve une expression exemplaire chez Sappho et dans sa légende ; on peut y ajouter encore celui du feu de la passion, certes déjà présent dans la deuxième strophe, où il décrit cependant le passé à la lumière du présent de la partie centrale ${ }^{48}$. En suivant une telle interprétation, ces strophes centrales évoquent, en lien au moins indirect avec Sappho, une passion élégiaque présente analogue à celle qui unit la Lydia de l'Ode 1.13 au rival du je lyrique; et si dans ce dernier poème, la passion élégiaque évoquait le mariage par l'espoir qu'elle dure éternellement, elle le fait ici en insistant sur la réciprocité amoureuse avec la métaphore du flambeau nuptial (face mutua). Dans ce contexte, je crois que Schwindt a raison de dire que ces clichés 
érotiques de l'élégie sont dépassés à la fin de l'ode, où est envisagé le retour à la prisca Venus après l'expérience élégiaque ${ }^{49}$. Or, ce retour est paradoxal: bien que les comparaisons hyperboliques, d'un point de vue érotique, présentent l'amant élégiaque comme préférable à l'amant lyrique (quamquam sidere pulcrior / ille est, tu leuior cortice et improbo / iracundior Hadria), la lyrique vise aussi une union pour la vie et jusqu'à la mort (tecum uiuere amem, tecum obeam lubens). Mais cette union lyrique n'implique pas la fidélité et la réciprocité amoureuses : la durabilité de cette relation, qui fait envisager la mort avec sérénité (lubens), est d'ordre non pas érotique, mais poétique, comme le montrent les hyperboles des deux strophes initiales, qui posent comme idéal la fortune (beatior) et surtout le renom (clarior) procurés par la poésie d'amour (multi Lydia nominis). Ainsi, le mariage est un idéal érotique pour l'élégie telle que vue par Horace, mais il représente pour sa lyrique l'éternité poétique qu'elle vise.

Ce dialogue entre lyrique et élégie, dans un chant amébée plein de traits érotiques et nuptiaux liés à Sappho, annonce ainsi la dialectique de l'ode 3.11 évoquée plus haut. Ce lien de proximité et ceux que j'ai relevés tout au long de cet article sont certainement signifiants. C'est pourquoi j'aimerais pour finir esquisser, à la lumière des réflexions présentées ci-dessus, une lecture linéaire centrée sur les indices de la présence du thème du mariage, de Vénus et de Sappho dans les Odes d'Horace.

\section{Sappho, Vénus, l'amour et le mariage dans la construction du recueil}

L'Ode 1.13, dans laquelle apparait pour la première fois le thème du mariage, est aussi le premier poème d'amour du recueil où l'on trouve des échos clairs de Sappho et où soit mentionnée Vénus ${ }^{50}$. Toutes deux sont toutefois déjà présentes plus tôt, sans y être directement liées à la poésie érotique. Dès la fin de l'ode initiale, on devine Sappho, avec Alcée, derrière le « luth de Lesbos » (1.1.34 : Lesboum... barbiton). Juste après, dans l' Ode 1.2, c'est en lien avec le thème politique des guerres civiles qu'est introduite la déesse Vénus, invoquée après Apollon et avant Mars et Mercure-Auguste parmi les divinités tutélaires de Rome, mais caractérisée par son empire érotique avec un premier écho de Sappho, le Désir volant autour de la déesse rappelant celui qui fait de même autour d'une jeune fille dans un fragment de la poétesse ; dans ce contexte nonérotique apparaît non seulement Vénus et un écho de Sappho, mais aussi la strophe sapphique, et ce poème introduit bien l'emploi tout sauf léger qu'Horace fait de ce rythme, et qui explique peut-être le qualificatif de mascula qu'il prête à la poétesse dans un texte célèbre (il faudra par contraste être attentif aux rares coïncidences de la strophe sapphique avec un thème érotique $)^{51}$. Vénus revient sur le devant de la scène aux Odes 1.3 et 1.4, mais respectivement comme protectrice de la navigation et divinité printanière qui guide le chœur des Grâces ; la déesse prépare certes par là l'arrivée du thème érotique dès l'ode suivante, mais ni elle ni Sappho ne sont directement présentes dans les premiers poèmes d'amour du recueil ${ }^{52}$. Paradoxalement, la toute première, $\mathrm{l}$ ' Ode 1.5, introduit même ce thème par la négative : elle prend en effet la forme d'une renuntiatio amoris, jugeant trompeuse l'idée qu'un amour puisse durer ${ }^{53}$. Si Vénus ne réapparait pas avant l'ode 1.13, Sappho est de nouveau évoquée par l'emploi nonérotique de la strophe sapphique dans les Odes 1.10 et 1.12, et dans ce dernier poème politique par un écho, à propos de l'éclat de la famille julienne, de fragments de Sappho comparant des jeunes filles à la lune qui brille plus que les astres alentour ${ }^{54}$. 

première reprise érotique, la première mention de Vénus en lien direct avec l'amour et, juste après une mise en garde - qui rappelle l'ode 1.5 - contre l'espoir qu'une passion douce-amère dure éternellement, la première célébration, dans sa strophe finale, de l'union éternelle. L'expression de cet absolu, dont j'ai montré plus haut qu'il était un idéal érotique inaccessible au je lyrique ainsi qu'une métaphore de l'éternité poétique qu'il recherche, précède de peu la première mention explicite du mariage à propos d'Hélène et de Pâris, dans l'ode 1.15, où Vénus apparaît du côté immoral, antimatrimonial et anti-lyrique, dont le destin est funeste ${ }^{55}$. À partir des deux poèmes érotiques qui suivent, les Odes 1.16 et 1.17 aussi liées à la figure d'Hélène, le thème du mariage n'apparaît plus explicitement au livre I, mais est parfois suggéré par des indices, allusions ou images évoquant soit une union durable dans le chant, comme dans la réécriture de Catulle et Sappho de l'ode 1.22 - le premier poème du recueil où le choix de la strophe sapphique coïncide avec un thème érotique -, soit l'âge de la vie propice à l'amour, comme à propos de Chloé dans l'ode 1.23 qui lui fait suite ${ }^{56}$. Quant à Vénus, dispensatrice de charmes qu'il faut rechercher, mais aussi de passions qu'il faut tempérer, sa duplicité persiste tout au long du livre, comme le montrent les trois odes mentionnant Glycère : si le sacrifice de l'ode 1.19 doit adoucir la brûlure qu'elle amène avec elle, la prière de l'Ode 1.30 - première ode érotique en strophes sapphiques où apparaisse Vénus avec un écho de Sappho - doit au contraire faire venir la déesse, et l'opposition entre ces deux rituels annonce peut-être les deux oxymores de l'ode 1.33, qui parle de la « non-douce Glycère » et de Vénus faisant des non-paires avec son joug ${ }^{57}$. Le livre I introduit donc progressivement le thème érotique en lien avec le jeu des échos de Sappho et des interventions de Vénus, en posant dès les Odes 1.13 (avec 1.22) et 1.15 le mariage comme un idéal, respectivement érotico-poétique et moral, mais en mettant surtout en scène - parfois avec quelques indices à connotation nuptiale - des amours non-matrimoniales.

odes érotiques du livre II se trouvent surtout dans sa première moitié, où alternent poèmes en strophes sapphiques et en strophes alcaïques, et sont annoncées à la fin de l'ode civique initiale par un retour au registre plus léger de Vénus ${ }^{58}$. Comme je l'ai montré plus haut, ces poèmes d'amour évoquent presque tous le mariage à propos de relations non-nuptiales: l'ode 2.4 est un épithalame ironique pour Xanthias et sa servante Phyllis; l'Ode 2.5 présente l'idéal érotique du mariage comme inaccessible au je, parce que le temps linéaire de l'amour et le temps cyclique de la poésie lyrique ne coïncident jamais; et dans l'ode 2.8, parodie de chant nuptial, même Vénus rit de la perfidie de Bariné, dont le comportement menace les mariages. Quant à la seconde moitié du livre, seuls ses trois premiers poèmes ont encore un lien avec la lyrique d'amour : l'ode 2.11 la définit comme explicitement symposiaque et non-matrimoniale en souhaitant que la courtisane Lydé arrive au banquet avec sa lyre; j'ai déjà montré que l'Ode 2.12 suggérait une union virtuelle, poétique, avec Licymnia, dont le mariage avec Mécène apparaît au contraire comme un amour élégiaque idéal ; enfin, l'Ode 2.13 évoque l'immortalité de la lyrique notamment érotique en montrant Sappho continuant de chanter ses plaintes amoureuses aux Enfers ${ }^{59}$.

31 Quant au livre III, il reprend et structure les différents aspects du mariage introduits dans les deux premiers livres. Tout d'abord, il amène l'idéal moral à son comble dans les odes romaines, avec les Odes 3.3 et 3.6 surtout. Ensuite, le premier poème après ce cycle, l'Ode 3.7, ramène des amours lyriques, non-matrimoniales, mais décrites avec des 
connotations nuptiales : Astérie pleure, séparée de Gygès jusqu'au printemps ; mais lui est un «jeune homme à la foi constante » et reste jusque-là sourd aux séductions de Chloé, qui brûle pour lui des mêmes feux qu'Astérie et se comporte comme les femmes mariées Sthénébée et Hippolyte face aux chastes Bellérophon et Pélée ; quant à Astérie, elle est appelée à résister au charme d'Énipée, décrit comme un prétendant digne d'être son fiancé ${ }^{60}$. Jouant sur les limites de la fidélité, ce poème annonce, par son double triangle amoureux, celui de l'ode 3.9. Cette dernière, dont j'ai montré plus haut qu'elle constitue un dialogue lyrico-élégiaque annonçant celui de l'ode 3.11, introduit même un cycle de quatre odes érotiques dont on peut dire qu'il définit, en lien plus ou moins explicite avec le mariage, les limites de la lyrique et de l'élégie ${ }^{61}$. L'Ode 3.10 parodie une situation typique de l'élégie, celle du chant de l'amant devant la porte close de l'aimée ( $\pi \alpha \rho \alpha \kappa \lambda \alpha u \sigma i ́ \theta v \rho o v)$, et donne un rôle anti-nuptial au je, qui irait jusqu'à s'opposer à un mari féroce et supplie Lycé de ne pas faire sa Pénélope ${ }^{62}$. Quant au dernier poème de ce petit cycle (Ode 3.12), il est purement lyrique : inspiré d'Alcée pour son mètre unique chez Horace et pour sa strophe initiale, ainsi que de Sappho pour la suivante, il décrit l'amour naissant de la jeune Néobulé pour le bel Hébrus, dont la splendeur et les qualités athlétiques feraient un fiancé idéal ${ }^{63}$.

Après l'adieu élégiaque d'Hypermestre à son mari à la fin de l'ode 3.11, l'élégie ne s'invite plus dans le discours lyrique. La thématique nuptiale réapparaît encore, dans l' Ode 3.15 pour présenter à nouveau les âges convenables ou non à l'amour, à propos d'une femme mariée qui se comporte comme sa propre fille, ou dans l'ode 3.24 pour réaffirmer l'idéal politique et moral du mariage en opposant la décadence romaine aux coutumes matrimoniales exemplaires des Scythes ${ }^{64}$. Mais il faut s'arrêter davantage sur le groupe des Odes 3.26-3.28, qui marquent l'adieu progressif à la lyrique, érotique en particulier. Par sa structure et son thème, l'ode 3.26 est la dernière ode érotique du recueil : cinquième poème avant la fin, cette renuntiatio amoris où le je dépose ses armes et sa lyre de poète d'amour dans le temple de Vénus est exactement symétrique à celle de l'ode 1.5, où il consacrait à Neptune ses vêtements éprouvés par la tempête érotique ; mais ce geste d'abandon est prolongé dans les deux poèmes suivants et ne se terminera que juste avant les deux épilogues solennels du recueil, dans l'ode 3.28 , où le je invite Lydé, dans un cadre de banquet champêtre, à répondre à ses chants et à réserver son dernier poème pour Vénus et pour la nuit ${ }^{65}$. C'est cependant l'ode 3.27 qui m'intéresse le plus dans ce groupe, et qui va m'amener à ma conclusion.

Véritable poème d'adieu, l'Ode 3.27 est un propemptikon adressé à Galatée, auquel est subordonné le récit de l'enlèvement d'Europe, occupé pour l'essentiel par le monologue désespéré de l'héroïne en Crète: entre Asie et Europe, entre rêve et réalité, entre innocence et culpabilité, entre piété à l'égard du père et passion pour l'être aimé, entre colère contre le taureau et honte d'elle-même, elle s'apprête à préférer la mort au statut d'esclave et de concubine en pays barbare quand Vénus finit par lui révéler son statut fortuné d'épouse de Jupiter et l'invite à l'accepter ${ }^{66}$. On note avec Michèle Lowrie que comme l'ode 3.11, ce poème présente un récit, lié au thème du mariage, qui n'est qu'en partie pertinent pour la situation décrite : Galatée, dont on comprend qu'elle part pour se marier, ne doit guère être rassurée par l'exemple d'Europe, qui se croit coupable d'amours illicites et se résout à mourir avant d'apprendre in extremis par une sorte de deus ex machina son mariage avec Jupiter ${ }^{67}$. Mais c'est de nouveau cette différence qui fait l'intérêt du poème. La crainte de l'héroïne reflète en effet moins l'état d'esprit de Galatée que celui du je lyrique au moment de la laisser partir et de dire 
adieu, à travers elle, à la lyrique notamment érotique dont il prend congé dans ce groupe de poèmes.

Or, en souhaitant à Galatée, et donc à sa poésie, d'être heureuse où qu'elle préfère aller et de vivre en préservant sa mémoire, Horace fait écho à un poème d'adieu de Sappho à une des jeunes filles de son groupe, qui part aussi se marier : il donne ainsi une nouvelle interprétation métaphorique du mariage, qui sépare certes le poète de sa poésie, mais, en unissant cette dernière à un lecteur, permet de perpétuer le souvenir de leur union $^{68}$. Pour amener le recueil à sa fin, l'ode 3.27 complète ainsi la définition lyrique du mariage proposée dans l'ode 3.11: alors qu'il y était la métaphore de cette éternité poétique visée par la lyrique, il devient ici celle de la réception durable de cette poésie, qui rend possible une telle éternité grâce à la mémoire. Horace souligne le lien entre l'adieu lyrique de l'Ode 3.27 et l'adieu élégiaque qui clôt l'ode 3.11, en faisant coïncider une dernière fois ici comme là thème érotique et strophe sapphique, écho de Sappho et apparition de Vénus.

\section{Conclusion}

$35 \mathrm{Au}$ terme de ce parcours rapide, qui mériterait d'être développé et illustré plus précisément dans une étude monographique, trois conclusions se dessinent. Premièrement, le mariage représente un idéal moral et participe, surtout dans des odes non-érotiques, de la célébration des valeurs traditionnelles romaines dont Auguste entreprend la restauration. Deuxièmement, cet idéal contraste manifestement avec le caractère non-nuptial de l'éros lyrique, lequel est toutefois atténué par des images nuptiales, notamment relatives à l'âge naturellement propice à l'amour: moment unique d'une vie amoureuse et en même temps rituel lyrique récurrent, le mariage, associé en particulier au cycle des saisons, confronte le temps linéaire de l'amour à celui, cyclique, de la poésie lyrique. Troisièmement, d'un point de vue métapoétique, le mariage ne représente pas comme dans l'élégie - telle que définie par Horace - l'espoir de faire durer à vie un lien érotique passionné, forcément exposé aux ruptures et aux plaintes, mais fonctionne plutôt comme métaphore de l'éternité promise par la lyrique.

Si Horace ne propose dans sa lyrique érotique ni épithalames - à part deux évocations indirectes du genre dans les Odes 2.4 et 2.8 -, ni échos directs des poèmes nuptiaux de Sappho - mais seulement des motifs traditionnels qu'elle est la première à employer -, c'est probablement parce que Catulle l'avait fait avant lui pour définir un idéal érotique de l'ordre de celui qu'Horace caractérise comme élégiaque. Le mariage est cependant un thème récurrent chez Horace. Mais en tant qu'idéal naturel, rituel et poétique, il ne s'y oppose pas aux amours non-matrimoniales de sa lyrique érotique, qui au contraire tendent vers lui comme vers une asymptote. Ainsi, alors que la réception de Sappho opposait éléments érotiques et nuptiaux, Horace rétablit une forme de continuité, non pas réelle comme dans le contexte du cercle de Sappho - qui voyait les jeunes filles y entrer pour être initiées à l'amour et en sortir pour se marier -, mais métaphorique, permettant de passer de la sphère de l'amour à celle du chant. Si l'idéal moral du mariage distingue en contexte politique des types de relations opposés, ce n'est pas le cas dans les odes érotiques, où le mariage n'en reste pas moins une métaphore essentielle à la définition de la poésie d'amour d'Horace et de sa lyrique en général, à la suite de Sappho et par contraste avec la tradition érotique latine de Catulle et des élégiaques. 


\section{BIBLIOGRAPHIE}

Acosta-Hughes 2006 : B. AcosTA-HUGHES, « Bucolic Singers of the Short Song : Lyric and Elegiac Resonances in Theocritus' Bucolic Idylls », in M. FANTUZzI et T. D. PAPANGHELIS (éd.), Brill's Companion to Greek And Latin Pastoral, Leiden/Boston, 25-52.

Ancona 1994 : R. ANConA, Time and the Erotic in Horace's Odes, Durham/London 1994.

Barchiesi 2000 : A. BARCHIESI, « Rituals in Ink : Horace on the Greek Lyric Tradition », in M. DEPEW et D. oBBinK (éd.), Matrices of Genre. Canons, Authors, and Society, Cambridge Mass. 2000, 167-182.

Biondi 1993 : G. G. BIONDI, « Catullo 'eolico' in Orazio lirico », in R. UGLIONE (éd.), Atti del Convegno Nazionale di Studi su Orazio : Torino, 13-14-15 aprile 1992, Torino 1993, 179-190.

Calame 1996 : C. CALAME, « Sappho's Group : An Initiation into Womanhood », in E. GREENE (éd.), Reading Sappho : Contemporary Approaches, Berkeley 1996, 113-124.

Cavallini 1986 : E. CAVALLINI, Presenza di Saffo e Alceo nella poesia greca fino ad Aristofane, Ferrara 1986.

Cucchiarelli 1999 : A. cucchiarelLI, « Hor. epist. 1, 19, 28 : pede mascula Sappho », Hermes 127, 1999, 328-344.

Cucchiarelli 2006 : A. CUCCHIARELLI, « La tempesta e il dio (forme editoriali nei Carmina di Orazio) », Dictynna 3, 2006, 73-136.

Davis 1991 : G. DAVIS, Polyhymnia. The Rhetoric of Horatian Lyric Discourse, Berkeley.

Giacomelli 1980 : A. GIACOMELLI, « The Justice of Aphrodite in Sappho Fr. 1 », TAPhA 110, 1980, 135142.

Habinek 1986 : Th. N. HABINEK, « The Marriageability of Maximus : Horace, Ode 4.1.13-20 », AJPh $107,1986,407-416$.

Heßen 2000 : B. HESSEN, « Liebe bis zum Tod? Bemerkungen zur letzten Strophe von Horaz, carm. $1,13 »$, inA. HALTENHOFF et F.-H. MUTSCHLER, Hortus litterarum antiquarum. Festschrift für Hans Armin Gärtner zum 70. Geburtstag, Heidelberg 2000, 243-251.

Hutchinson 2001 : G. O. HUTCHINSON, Greek Lyric Poetry : A Commentary on Selected Larger Pieces, Oxford 2001.

Lowrie 1995 : M. LOWRIE, « A Parade of Lyric Predecessors : Horace C. 1.12-1.18 », Phoenix 49, 1995, 33-48.

Lowrie 1997 : M. LowRIE, Horace's Narrative Odes, Oxford 1997.

Lyne 1995 : R. O. A. M. LYNE, Horace : Behind the Public Poetry, New Haven 1995.

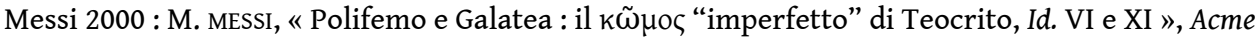
$53.1,2000$, p. 23-41.

Most 1995 : G. W. MOST, « Reflecting Sappho », BICS 40, 1995, 15-38.

Nagy 1994 : G. NAGY, « Copies and Models in Horace Odes 4.1 and 4.2 », CW 87, 1994, 415-426.

Nisbet \& Hubbard 1970 : R. G. M. NISBET et M. HUBBARD, A Commentary on Horace : Odes, Book I, Oxford 1970. 
Nisbet \& Hubbard 1978 : R. G. M. NISBET et M. HUBBARD, A Commentary on Horace: Odes, Book II, Oxford 1978.

Nisbet \& Rudd 2004 : R. G. M. NISBET et N. RUDD, A Commentary on Horace : Odes, Book III, Oxford 2004.

Putnam 1977 : M. C. J. PUTNAM, « Horace Odes 3.9 : The Dialectics of Desire », in J. H. D'ARMS et J. W. EADIE (éd.), Ancient and Modern : Essays in Honor of Gerald F. Else, Ann Arbor 1977, 139-157.

Putnam 1986 : M. C. J. PUTNAM, Artifices of Eternity. Horace's Fourth Book of Odes, Ithaca/London 1986.

Putnam 2006 : M. C. J. PUTNAM, Poetic Interplay. Catullus and Horace, Princeton/Oxford 2006.

Santirocco 1986 : M. S. SANTIRocco, Unity and Design in Horace's Odes, Chapel Hill (NC) 1986.

Schwindt 2002 : J. P. SCHWINDT, « Die leichte und die schwere Muse. Über einige Gesichtspunkte der Erklärung von Horaz c. 3, 9 », Gymnasium 109, 2002, 497-517.

Segal 1973 : Ch. P. SEGAL, « Felices ter et amplius. Horace, Odes, I. 13 », Latomus 32, 1973, 39-46.

Syndikus 2001 : H. P. Syndikus, Die Lyrik des Horaz. Eine Interpretation der Oden, II : Drittes und viertes Buch, Darmstadt ${ }^{3} 2001\left({ }^{1} 1973\right)$.

Tarrant 1995 : R. J. TARRANT, « Da Capo Structures in Some Odes of Horace », in S. J. HARRISON (éd.), Homage to Horace, Oxford 1995, 32-49.

Thévenaz 2003 : O. THÉVENAZ, « Poétiques comparées : de l'Aphrodite de Sappho à la Vénus d'Horace ", in U. HEIDMANN (éd.), Poétiques comparées des mythes - De l'Antiquité à la Modernité. En hommage à Claude Calame = Études de lettres 3, Lausanne 2003, 107-127.

Thévenaz 2004 : O. THÉVENAZ, « Fleurs d'intertextualité : images épithalamiques chez Sappho, Catulle et Virgile ", in Ph. MUDRY et O. THÉVENAZ (éd.), Nova studia Latina Lausannensia, Études de lettres 1-2, Lausanne 2004, 57-78.

West 1995 : D. WEST, « Reading the Metre in Horace, Odes 3.9 », in S. J. HARRISON (éd.), Homage to Horace, Oxford 1995, 100-107.

Woodman 2002 : T. WOODMAN, «Biformis Vates: The Odes, Catullus and Greek Lyric », in T. WoodMAN et D. FEENEY (éd.), Traditions and Contexts in the Poetry of Horace, Cambridge 2002, 53-64.

Yatromanolakis 1999 : Dimitrios YATROMANOLAKIS, « Alexandrian Sappho Revisited », HSCPh 99, 1999, 179-195.

\section{NOTES}

1. Cet article s'inscrit en marge d'une thèse de doctorat dirigée par le Prof. Philippe Mudry et intitulée Sappho à Rome. Discours amoureux en dialogue, projet soutenu pour l'année académique 2006-2007 par la Fondation Théodore Lagonico, la Société académique vaudoise et la Fondation Me J.-J. Van Walsem pro Universitate. Il résulte de l'élaboration de trois exposés tenus pendant l'année académique 2005-2006, respectivement à l'Université de Neuchâtel lors d'une séance du Groupe Romand des Études Grecques et Latines, à l'Université de Genève dans le cadre du séminaire du Prof. Damien Nelis consacré aux Odes d'Horace, et à l'Université Charles-de-Gaulle Lille 3 dans le cadre du séminaire mensuel de l'équipe de recherche UMR CNRS 8143 HALMAIPEL, à laquelle j'ai été généreusement associé pour ladite année, financée par un subside du Fonds National Suisse de la recherche scientifique. Je tiens à remercier de leurs remarques les participants à ces différentes manifestations, et tout spécialement les Prof. Alain Deremetz et 
Jacqueline Fabre-Serris pour les excellents échanges que j'ai eus avec eux et pour leur invitation à publier ce travail dans la revue Dictynna.

2. Pour la nature du groupe de jeunes filles de Sappho, voir par exemple la présentation synthétique de Calame 1996, avec le résumé nuancé donné par Hutchinson 2001 :144-146.

3. On peut trouver un indice de la circulation distincte des poèmes nuptiaux de Sappho dans le fait que le traité Sur le style du Ps.-Démétrios de Phalère ne cite de Sappho que des fragments de

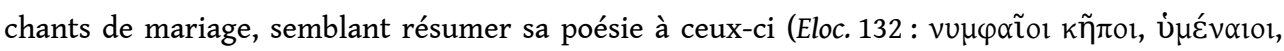

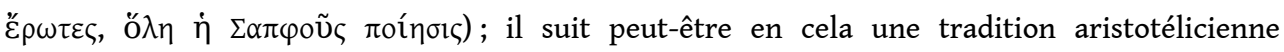
antérieure à la constitution du recueil alexandrin de Sappho (Théophraste). Pour la reprise des poèmes érotiques de Sappho au banquet, voir p. ex. Most $1995: 31$ et n. 81, qui cite quelques textes l'attestant et évoque des représentations de Sappho au banquet sur des vases de l'époque classique ; les textes discutés par Cavallini 1986 sont aussi liés assez étroitement au banquet.

4. Dans sa discussion de la Sappho alexandrine, Yatromanolakis 1999 avance l'hypothèse convaincante de recueils partiels, de poèmes nuptiaux en particulier; mais je ne partage pas sa préférence pour une édition en huit livres.

5. Pour les échos de la Sappho érotique chez Théocrite, voir en dernier lieu Acosta-Hughes 2006, en particulier 37-39 et 51-52, avec références primaires et secondaires ; pour la Sappho nuptiale dans le Cyclope (Theoc. 11), voir Messi 2000, qui exagère cependant le sérieux de ce chant et néglige les échos des poèmes d'amour malheureux de Sappho.

6. Pour des références et plus de détails sur mes réflexions à ce sujet, voir Thévenaz 2004.

7. Woodman 2002, notamment, montre que Sappho apparaît dans l'ode à la lyre d'Alcée (1.32, en strophes sapphiques, avec un écho aux v. 3-4: age, dic Latinum, / barbite, carmen, cf. Sapph.

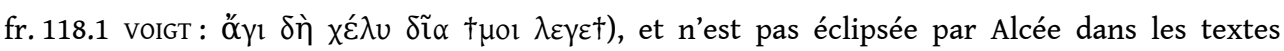
d'Horace qui semblent le faire primer sur elle (carm. 2.13.21-32, epist. 1.19.23-33; elle est même bien plus en évidence que lui en carm. 4.9.5-12). Pour le rapport d'Horace à Catulle, voir Putnam 2006. Les traductions des Odes d'Horace que je donne dans la suite de cet article sont celles, parfois légèrement retouchées, de François Villeneuve parues aux Belles Lettres (CUF), Paris ${ }^{1} 1929$.

8. Hor. carm. 4.1.3-5: non sum qualis eram bonae / sub regno Cinarae. desine, dulcium/ mater saeua Cupidinum... ; cette apostrophe à Vénus est composée du vers initial de l'Ode 1.19 (mater saeua Cupidinum) précédé de l'adjectif dulcium, qui s'oppose à saeua en un oxymore rappelant celui du

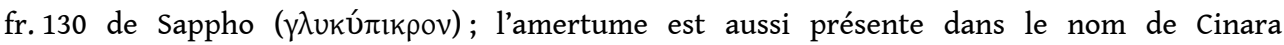
(« artichaut»), qui n'apparaît certes pas dans le premier recueil, mais est cité comme celui d'une ancienne amante en epist. 1.7.28 et 1.14.33, et encore en carm. 4.13.21-22.

9. Hor. carm. 4.1.33-36: sed cur... / manat rara mea lacrima per genas ? / cur... / inter uerba cadit

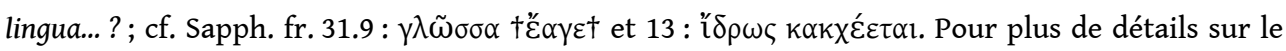
rapport entre l'Ode 4.1 d'Horace et le fr. 1 de Sappho, je me permets de renvoyer à l'article que je lui ai consacré (Thévenaz 2003) à la suite de Putnam 1986 : 33-47, Nagy 1994 et Barchiesi 2000 (en particulier 170-173); on notera en particulier la présence du verbe performatif de la prière au

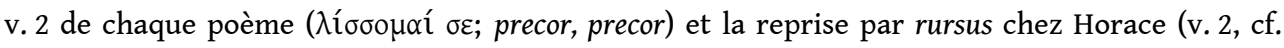
v. 8 : reuocant) du triple $\delta$ ữ $\tau \varepsilon$ de Sappho (v. 15, 16, 18), ainsi que le contraste entre $\varepsilon \lambda^{\prime} \theta \varepsilon$ chez Sappho (v. 5 et 25) et abi chez Horace (v. 7); si Aphrodite est appelée à combattre aux côtés de Sappho (v. $28: \sigma u ́ \mu \mu \alpha \chi \circ \varsigma$ ह̌ $\sigma \sigma o)$, elle mène la guerre contre Horace (v. 2 : bella moues).

10. Pour la recusatio, voir la mise au point de Lyne $1995: 31-39$, ainsi que les observations de Davis 1991 : 28-79 (en particulier 65-71 sur l'Ode 4.1), qui montre comment Horace l'utilise comme une forme de prétérition intégrant des éléments du genre qu'il prétend refuser.

11. Voir Habinek 1986 (avec références), qui montre en particulier que l'éloge de Paulus Maximus (4.1.9-20) est celui d'un futur époux et note aussi cette opposition entre deux formes d'éros antithétiques; parmi les traits élégiaques de l'amour du jeune Maximus, je citerai les blandae preces (v. 8), la militia de l'amour (v. 16) et la mention d'un rival dont il triomphera (v. 18). Pour la 
nature symposiaque de l'éros du je lyrique, Hor. carm. 4.1.29-32: me nec femina nec puer/iam nec spes animi credula mutui / nec certare iuuat mero / nec uincire nouis tempora floribus (on notera aussi l'incrédulité face à la réciprocité amoureuse).

12. Les leges Iuliae sur les adultères et les mariages sont évoquées en Hor. carm.4.5.21-25 et 4.15.10-12 (ainsi que carm. saec. 13-20) ; mais ce sont des relations spécifiquement non-maritales que décrit la lyrique érotique d'Horace en général (voir par exemple Ancona 1994), et aussi les poèmes d'amour du livre IV en particulier : outre le rappel de Cinara en 4.1.4 et 4.13.21-22, les Odes 4.11 et 4.13 s'adressent aux courtisanes Phyllis et Lycé, et 4.10, comme la fin de 4.1, au jeune éphèbe Ligurinus. Le poème d'ouverture, qui cherche à remplacer l'amour non-matrimonial frappant le je lyrique à contretemps par un mariage et par l'annonce à la déesse que le futur époux instituera un rituel en son honneur, est interprété par Putnam 1986: 40 comme « a prayer for the reritualization of Venus ".

13. Hor. carm. 3.6.17-32 : fecunda culpae saecula nuptias | primum inquinauere et genus et domos | [...]. || motus doceri gaudet Ionicos / matura uirgo et fingitur artibus, / iam nunc et incestos amores / de tenero meditatur ungui. || mox iuniores quaerit adulteros / inter mariti uina, neque eligit / cui donet inpermissa raptim / gaudia luminibus remotis, || sed iussa coram non sine conscio / surgit marito, seu uocat institor / seu nauis Hispanae magister, / dedecorum pretiosus emptor. Dans la partie finale décrivant le déclin des générations depuis les guerres puniques, voir en particulier les v. 33-34 : non his iuuentus orta parentibus / infecit aequor sanguine Punico..., et 46-48: aetas parentum [...] tulit / nos nequiores, mox daturos / progeniem uitiosiorem.

14. Je signale ici les autres : en 3.5.41-43, Régulus, dont l'exemple prépare l'évocation idéale de la génération des guerres puniques dans l'Ode 3.6 (voir note précédente), va jusqu'à écarter «le baiser de sa chaste épouse et ses jeunes enfants ", parce qu'en sacrifiant sa liberté pour celle de sa patrie, il perd son rang de citoyen romain; en 3.14.5, Livie est dite unico gaudens mulier marito, dans une déformation de la vérité qui sert les intérêts politiques et moraux de son mari ; et en 3.24.17-24, les traditions matrimoniales des Scythes sont décrites comme exemplaires en regard de la décadence morale romaine.

15. Hor. carm. 3.3.16-68, en particulier 19-20 : fatalis incestusque iudex / et mulier peregrina, et 64 : coniuge me Iouis et sorore.

16. Hor. carm. 1.15, en particulier 5-7: mala ducis aui domum/ quam multo repetet Graecia milite, I coniurata tuas rumpere nuptias, ainsi que 16-20: nequicquam thalamo grauis / hastas [...]/ uitabis strepitumque et celerem sequi / Aiacem : tamen, heu serus, adulteros / crines puluere collines.

17. Hor. carm. 1.15.14-15: grataque feminis / inbelli cithara carmina diuides ; 29-32: quem [Tydiden] tu, ceruus uti uallis in altera / uisum parte lupum graminis inmemor, / sublimi fugies mollis anhelitu, / non hoc pollicitus tuae; ce rappel des promesses à Hélène permet de rapprocher cette comparaison des images animales non seulement épiques, mais aussi nuptiales, dont elle constitue le négatif (voir infra et n. 22, 27 et 29 pour d'autres images de ce type ; par contraste, Sappho compare le fiancé à Achille ; cf. test. 218 voIGT). Pour l'interprétation métapoétique exposée ici, voir Davis 1991 : 2328 et Lowrie $1997: 123-137$, en particulier 133-135.

18. Hor. carm. 1.17.18-20 : ... fide Teia / dices laborantis in uno / Penelopen uitreamque Circen. Ces vers montrent que la lyrique, représentée ici par Anacréon de Téos, joue sur la limite que fixe le mariage; c'est peut-être aussi le cas au poème érotique précédent, commençant par une hyperbole (Hor. carm.1.16.1: o matre pulchra filia pulchrior) qui rappelle celle d'un fragment

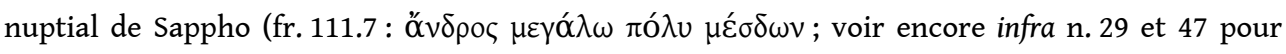
d'autres hyperboles nuptiales). Sur ce groupe d'odes, voir en dernier lieu Putnam 2006.

19. Hor. carm. 1.13.17-18: felices ter et amplius / quos inrupta tenet copula... ; voir infra et n. 41.

20. Hor. carm. 2.12, en particulier 13-20 pour l'éloge assumé par le je lyrique : me dulcis dominae Musa Licymniae / cantus, me uoluit dicere lucidum / fulgentis oculos et bene mutuis / fidum pectus amoribus; |l quam nec ferre pedem dedecuit choris / nec certare ioco nec dare bracchia / ludentem nitidis uirginibus sacro/ Dianae celebris die; et 21-28 pour l'éloge élégiaque dans la perspective de 
Mécène: num tu quae tenuit diues Achaemenes / [...]/ permutare uelis crine Licymniae, / [...] /| cum flagrantia detorquet ad oscula / ceruicem aut facili saeuitia negat / quae poscente magis gaudeat eripi, I interdum rapere occupet.

21. Hor. carm. 2.4.13-22 : nescias an te generum beati / Phyllidis flauae decorent parentes... | [...]. || crede non illam tibi de scelesta / plebe delectam, neque sic fidelem, / sic lucro auersam potuisse nasci / matre pudenda. || bracchia et uoltum teretisque suras / integer laudo... Pour la déclaration de vertu du laudateur, reprise ici non sans ironie par Horace, voir le conseil de Men. Rhet. 3.404.11-14 SPENGEL

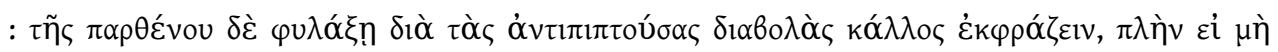

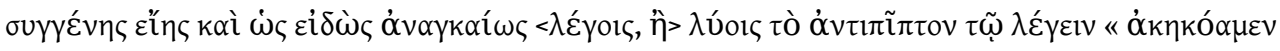
$\tau \alpha \tilde{\tau} \tau \alpha »$.

22. Hor. carm. 2.8.5-8 : ... simul obligasti / perfidum uotis caput, enitescis / pulchrior multo iunenumque prodis / publica cura, 21-24: te suis matres metuunt iuuencis, / te senes parci miseraeque nuper / uirgines nuptae, tua ne retardet / aura maritos; la critique a noté que cette dernière phrase parodiait une strophe de l'éloge d'Hymen dans le premier épithalame de Catulle (61.51-55: te suis tremulus parens / inuocat, tibi uirgines | zonula soluunt sinus, / te timens cupida nouos / captat aure maritus ; voir Ancona 1994 : 82-85, et en dernier lieu Putnam 2006 : 128-131, avec références) ; on peut ajouter que le faux serment du début prend ainsi un caractère anti-nuptial.

23. Hor. carm. 2.5.1-12 : nondum subacta ferre iugum ualet / ceruice, nondum munia comparis / aequare nec tauri ruentis | in uenerem tolerare pondus. || circa uirentis est animus tuae | campos iunencae [...] | ludere cum uitulis [...]/ praegestientis. tolle cupidinem/ inmitis uuae: iam tibi liuidos/ distinguet autumnus racemos / purpureo uarius colore; pour des comparaisons avec une génisse et du raisin vert dans un contexte nuptial, voir l'adresse de Polyphème à Galatée chez Théocrite (11.20 :

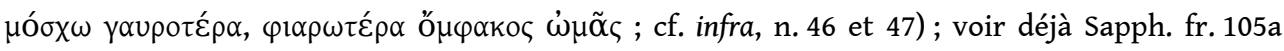
pour une comparaison nuptiale avec un fruit, élément qui devient ensuite traditionnel.

24. Hor. carm. 2.5.13-16: iam te sequetur; currit enim ferox / aetas et illi quos tibi dempserit / adponet annos ; iam proterua / fronte petet Lalage maritum...

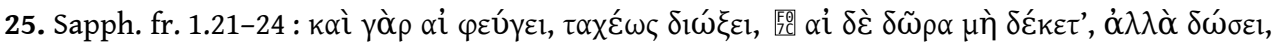

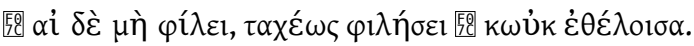

26. Dans l'Ode 2.5 d'Horace sont répétés tour à tour les adverbes nondum (v. 1 et 2), nunc (v. 6 et 7)

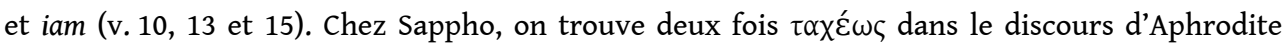
(n. 25) ; cet indice de temps linéaire, qui s'oppose au triple $\delta \eta \tilde{u} \tau \varepsilon$ cyclique référé précédemment au je lyrique $(\mathrm{v} .15,16,18)$, plaide pour la thèse selon laquelle la jeune fille ne répondra pas à l'amour de Sappho, mais sera à son tour amoureuse (Giacomelli 1980).

27. Hor. carm. 1.33, en particulier 10-12 : sic uisum Veneri, cui placet imparis / formas atque animas sub iuga aenea / saeuo mittere cum ioco. Juste avant, la résistance de Pholoé à l'adultère (v. 9 : adultero) arrête la chaîne d'amours non-réciproques censée montrer à Tibulle que la trahison de Glycère ne fait pas de lui une exception. Le poème se clôt sur un exemple positif de joug inégal, celui du je lui-même, qui préfère à une relation nuptiale les chaînes d'une affranchie (v. 13-15 : ipsum me melior cum peteret Venus, I grata detinuit compede Myrtale / libertina...).

28. Hor. carm. 1.23, en particulier 11-12: tandem desine matrem/ tempestiua sequi uiro; cf.

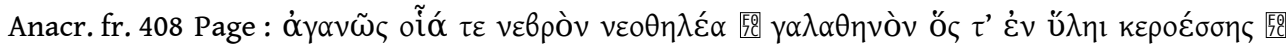

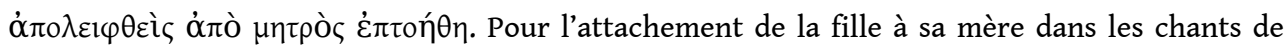
mariage, voir Catull. 61.56-59 et 62.21-23, et Sapph. fr. 104a (avec des comparaisons animales); dans le fr. 105b, la fleur d'hyacinthe, à laquelle est comparée la jeune fille qui perd sa virginité, est piétinée par des bergers.

29. Hor. carm. 1.36, en particulier 18-20 : ... nec Damalis nouo/ diuelletur adultero / lasciuis hederis ambitiosior. Les comparaisons végétales sont présentes dans les poèmes nuptiaux de Sappho (fr. 105b, 115), et reprises notamment par Catulle (61.34-35, 106-109 et passim, 62.39-54; voir Thévenaz 2004 avec références). On trouve déjà plus tôt dans le livre I d'autres comparaisons 
hyperboliques (1.16.1 : voir supra n. $17 ; 1.19 .6$ : splendentis Pario marmore purius, et - en négatif 1.33.15-16 : fretis acrior Hadriae / curuantis Calabros sinus), mais leur caractère nuptial apparait ici l'image végétale, et surtout dans l'accumulation de l'Ode 3.9 ; voir infra et n. 46 et 47.

30. Hor. carm. 3.11, en particulier 9-12: quae uelut latis equa trima campis / ludit exultim metuitque

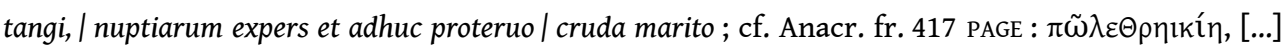

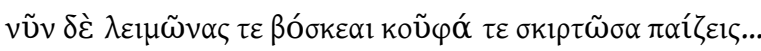

31. Lowrie 1997 : 275-297 (en particulier 282, avec n. 40, et 296) ; pour plus de détails sur cette hypothèse, voir infra.

32. Hor. carm. 3.11.25-52, en particulier 33-36 et 37-52 pour l'histoire d'Hypermestre et son discours à Lyncée ; pour la structure du poème, voir Lowrie $1997: 289-292$.

33. Hor. carm. 3.11.3-8 : tuque testudo resonare septem / callida neruis, / nec loquax olim neque grata, nunc et / diuitum mensis et amica templis, / dic modos, Lyde quibus obstinatas / applicet auris ; cf. Sapph.

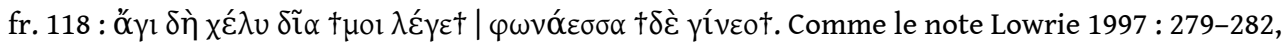
non seulement Lydé doit deux fois écouter (v. 9 et 25), mais elle est associée à la lyre en tant que destinataire réelle du poème, ainsi que par son nom, qui fait penser à lyra et évoque le mode lydien ; tout cela en fait une figure du public et de la poésie.

34. Hor. carm. 3.11.33-42: una de multis face nuptiali / digna periurum fuit in parentem/ splendide mendax et in omne uirgo | nobilis aeuom, || « Surge », quae dixit iuueni marito / «[...] socerum et scelestas | falle sorores, / quae uelut nactae uitulos leaenae / singulos eheu lacerant [...]». Lowrie 1997: 281 souligne que la comparaison animale négative des Danaïdes criminelles est un contre-exemple pour Lydé, elle-même objet d'une comparaison de ce type.

35. Hor. carm. 3.11.13-24 pour la catabase de la lyre d'Orphée et, pour l'inscription plaintive compensant l'interruption du mariage d'Hypermestre (soulignée par le contraste, dans le texte cité supra n. 33, entre uirgo et marito), 50-52: « [...] i secundo / omine et nostri memorem sepulcro / scalpe querellam. », avec Lowrie 1997 : 282-297, qui appuie ensuite (297-316) son interprétation en analysant l'ode 3.27, que je reprendrai en fin d'article (infra et n. 66-68).

36. Lowrie $1997: 277$ n. 24.

37. Pour le regard extérieur, voir notamment Hor. carm. 1.13.7 : arguens ; pour le rapport à Sappho, je peux me limiter à renvoyer au commentaire de Nisbet \& Hubbard 1970: 169-170, qui mettent en évidence en particulier dans les trois premières strophes le caractère épigrammatique auquel je me réfère plus loin.

38. Hor. carm. 1.13.1-3: Cum tu, Lydia, Telephi / ceruicem roseam, cerea Telephi / laudas bracchia, uae... ; Catull.51.1-2: Ille mi par esse deo uidetur, | ille, si fas est, superare diuos...; Sapph. fr. 31.1-2:

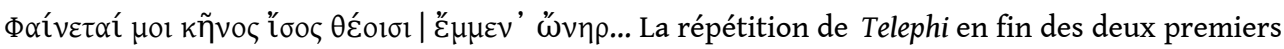
vers chez Horace répond à celle de ille au début des deux vers initiaux du poème de Catulle, insistance qui ne correspond à rien chez Sappho. Horace en fait un poème de jalousie, en nommant cette tierce personne, qui fait l'objet d'un éloge du tu et non du je, et en court-

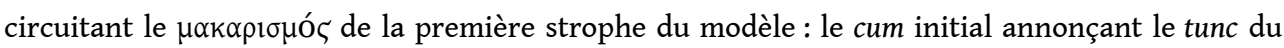
v. 5 reprend la corrélation temporelle de la deuxième strophe du poème de Sappho (fr. $31.7: \omega \dot{\omega} \varsigma .$.

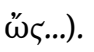

39. Hor. carm. 1.13.5: tunc nec mens mihi... reprend Catull. 51.5-12: ... misero quod omnis / eripit sensus mihi..., alors que 1.13.5-7: ... nec color / certa sede manet, umor et in genas / furtim labitur

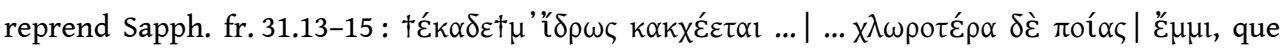
Catulle n'avait pas traduit. On signalera aussi que 1.13.9: uror rappelle le désir brûlant du fr. 48

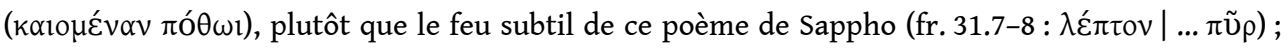
voir encore note suivante.

40. Hor. carm. 1.13.13-15: non, si me satis audias, / speres perpetuum dulcia barbare / laedentem oscula ; on notera d'une part que l'oxymore évoquant cette passion rappelle l'amour doux-amer de 


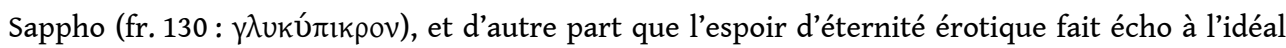
de Catulle (109.6, et déjà 1 : perpetuum).

41. Hor. carm. 1.13.17-20: felices ter et amplius / quos inrupta tenet copula, nec malis / diuolsus querimoniis / suprema citius soluet amor die. Ces vœux rappellent ceux qui terminent les épithalames

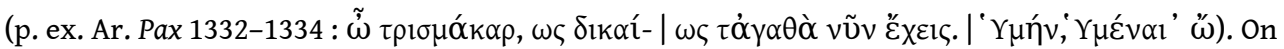
notera que cette bénédiction nuptiale finale indique peut-être qu'Horace considère, comme une partie de la critique moderne, que l'homme célébré par Sappho au début de son poème et assis face à la jeune fille aimée est le probable futur mari de cette dernière.

42. Voir Heßen 2000 pour l'historique de cette question, et Segal 1973 pour une interprétation sensible et nuancée.

43. Les échos de Catulle sont signalés entre autres par le commentaire de Nisbet \& Hubbard 1970 ad loc., et le rapport entre l'Ode 1.22 d'Horace et le poème 11 de Catulle étudié plus en détail par Biondi 1993. Si l'Ode 1.13 présentait, comme le poème 11 de Catulle, une structure asymétrique et des changements de ton aux deux dernières strophes, l'Ode 1.22 rétablit une structure symétrique en parfait da capo (Tarrant 1995 : 36 n. 5 pour cette ode) ; ainsi, l'anaphore de siue / seu qui s'étend sur les quatre premières strophes du poème 11 de Catulle est répartie ici en une de siue... siue... au début (v. 5-6) et une de pone... pone... qui y répond à la fin (v. 17 et 21).

44. Hor. carm. 1.22.23-24: dulce ridentem Lalagen amabo, / dulce loquentem; cf. Sapph. fr. 31.3-5:

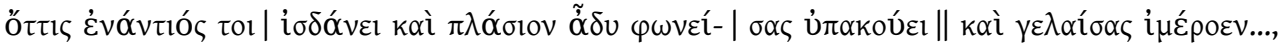
avec Catull. 51.3-5 : qui sedens aduersus identidem / te spectat et audit dulce ridentem...

45. Schwindt 2002 (510-515 pour la discussion des parallèles textuels), ainsi que Tarrant 1995 : 46-49 pour la structure faussement limpide de cette unique ode en da capo du livre III ; pour les subtilités métriques en particulier, voir West 1995 ; pour les éléments de commentaire, je me contente de renvoyer à Nisbet \& Rudd 2004 ad loc., qui font brièvement mention (133) du modèle nuptial (un peu plus de détails chez Syndikus $2001: 105-107$ et n. 8, 9 et 18).

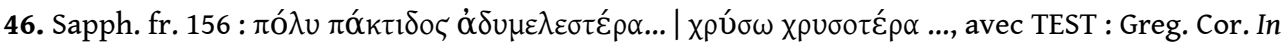

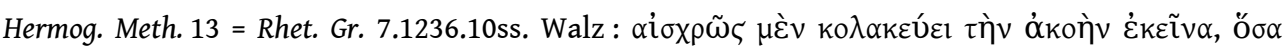

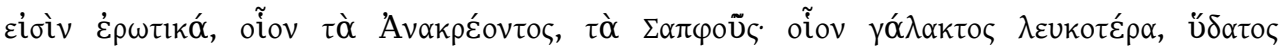

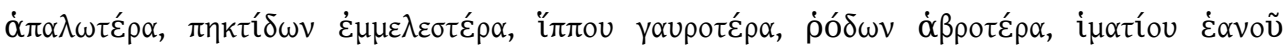

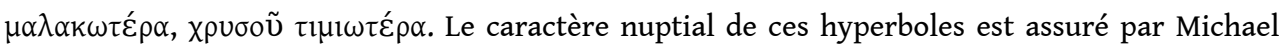

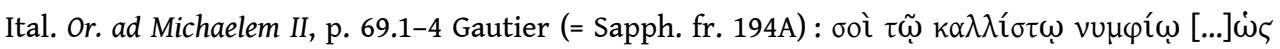

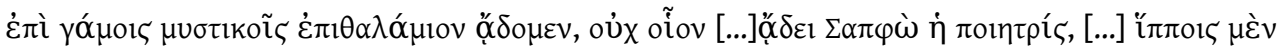

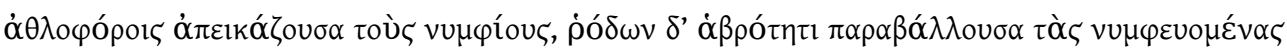

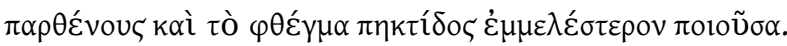

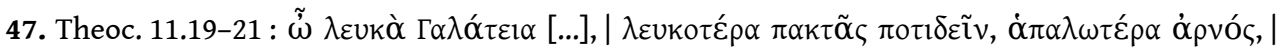

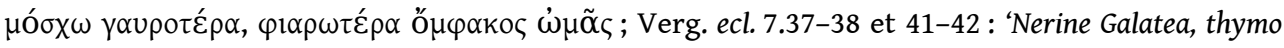
mihi dulcior Hyblae, / candidior cycnis, hedera formosior alba...' ; 'immo ego Sardoniis uidear tibi amarior herbis / horridior rusco, proiecta uilior alga...'. On trouve déjà de telles hyperboles, aussi bien positives que négatives, au livre I des Odes d'Horace (voir supra n. 29), et encore deux négatives dans les odes érotiques entourant celle qui nous intéresse ici (3.7.21 : scopulis surdior Icari, et, sous forme de litote, 3.10.17-18: nec rigida mollior aesculo / nec Mauris animum mitior anguibus), mais l' Ode 3.9 les accumule d'une façon qui rappelle davantage cette tradition.

48. Pour le lien entre la maîtrise des poèmes de Sappho et le portrait de la puella docta néotérique et élégiaque, voir p. ex. Phld. AP 5.132., Catull. 35.16-17, Ou. Ars 3.331; pour le motif de la mort amoureuse, on pensera d'une part aux poèmes de Sappho qui évoquent la mort (p. ex. fr. 31.15, 94.1 et 95.10-13), d'autre part au légendaire suicide de Sappho du promontoire de Leucade (voir notamment Ou. epist. 15) ; pour le motif du feu - Lydia répond au v. 13 par me torret au arsisti du v. 6, qui se réfère plutôt au feu qui brûle maintenant son interlocuteur qu'au passé qui était

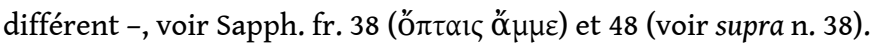


49. Schwindt 2002 : 515-517 (et 506-507 pour la temporalité complexe de ce poème où passé et futur sont vus à travers le présent, mais l'éclipsent), ainsi que Putnam 1977 pour la dialectique érotique.

50. Hor. carm. 1.13.15-16:... oscula, quae Venus / quinta parte sui nectaris imbuit.

51. Vénus est invoquée pour la première fois après Apollon et avant Mars et Mercure-Auguste en Hor. carm.1.2.33-34 où elle est dite Erycina ridens / quam Iocus circumuolat et Cupido, malgré sa fonction de garante de l'empire de Rome; le participe ridens évoque peut-être le sourire

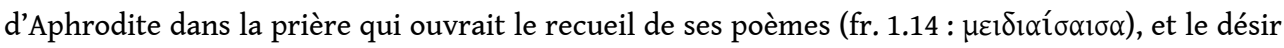
qui vole autour de Vénus rappelle plus clairement celui qui fait de même autour d'une jeune fille

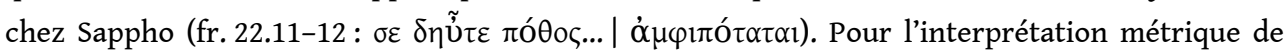
(pede) mascula Sappho (Hor. epist. 1.19.28), voir Cucchiarelli 1999.

52. Vénus apparaît en 1.3.1 comme protectrice de la navigation, en 1.4.5-6 comme chorège des Nymphes et des Grâces, mais ni elle ni Sappho n'est présente en 1.5, premier poème érotique du livre, ni dans la première ode à Lydie (1.8), ni dans les autres passages liés au thème de l'amour (1.6.17-20, 1.9.15-24, 1.11).

53. Hor. carm. 1.5, en particulier 10-12 : qui semper uacuam, semper amabilem / sperat, nescius aurae / fallacis.

54. Hor. carm. 1.12.46-48: micat inter omnis / Iulium sidus, uelut inter ignis / luna minores, avec Sapph.

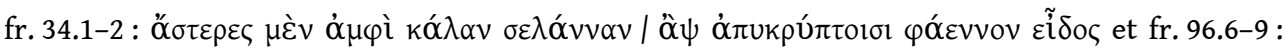

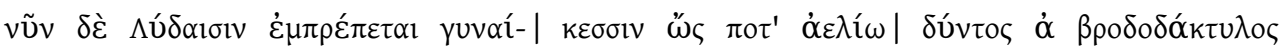

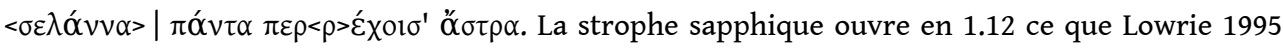
appelle « A Parade of Lyric Predecessors ", après avoir marqué en 1.10, par son retour après 1.2, la fin des "Paradeoden", c'est-à-dire des neuf premiers poèmes du recueil de mètres tous différents; pour une étude récente de la structure du recueil des Odes mettant en évidence notamment des groupes de dix (1.10, 1.20 et 1.30 sont notamment en strophes sapphiques), voir Cucchiarelli 2006.

55. Hor. carm. 1.15.13 : nequiquam Veneris praesidio ferox... ; pour le reste, voir supra et n. 16-17.

56. Pour ces différents poèmes, voir supra et $n .18,28$ et 44 . En plus des autres poèmes érotiques non-matrimoniaux cités à la note suivante, on peut ajouter l'ode 1.25 (v. 9 : moechos), variation sarcastique sur le $\pi \alpha \rho \alpha \kappa \lambda \alpha v \sigma i ́ \theta v \rho o v$, et rappeler à la fin du livre l'ode 1.36, qui décrit un amour adultère par une double image nuptiale (voir supra n. 29).

57. Hor. carm. 1.19, en particulier 16 : mactata ueniet lenior hostia, ainsi que 5-7: urit me Glycerae nitor [...], / urit grata proteruitas, qui rappelle le motif du feu érotique présent chez Sappho (voir supra n. 39 et 48 ) ; le petit hymne de l'Ode 1.30 rappelle ceux de Sappho (1-4:o Venus regina Cnidi Paphique, / sperne dilectam Cypron et uocantis / ture te multo Glycerae decoram / transfer in aedem, cf.

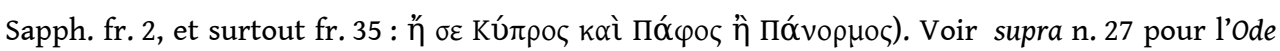
1.33 (v. 2 : inmitis Glycerae, cf. l'amour doux-amer de Sapph. fr. 130).

58. Hor. carm. 2.1.39-40 : mecum Dionaeo sub antro / quaere modos leuiore plectro; de fait, le livre ne présente par la suite plus de poème dont le thème principal soit politique, mais beaucoup d'odes, morales et érotiques, liées au contexte du banquet. Pour la structure du livre II, voir Nisbet \& Hubbard 1978 : 1-6 et Santirocco $1986: 83-95$, qui fait aller la première partie du livre au-delà de la moitié (Ode 2.10) jusqu'à l'ode 2.12.

59. Hor. carm. 2.11.21-23: quis deuium scortum eliciet domo / Lyden? Eburna dic, age, cum lyra/ maturet ; pour Sappho chantant aux Enfers, voir 2.13.21-28; pour l'Ode 2.12, voir supra et n. 19.

60. Hor. carm. 3.7, en particulier 4 : constantis iuuenem fide, 13-20 pour la référence aux épouses adultères du mythe et 25-28 pour les qualités notamment athlétiques insurpassables d'Énipée.

61. Pour le groupe des Odes 3.10-3.12, annoncées par 3.7 et reprises par 3.15, voir Santirocco $1986: 125-128$. 
62. Hor. carm. 3.10, en particulier 2 : saeuo nupta uiro ; 11-12: non te Penelopen difficilem procis / Tyrrhenus genuit parens (voir encore supra n. 47 pour les hyperboles anti-nuptiales de la fin du poème).

63. Hor. carm. 3.12; on rapproche ce poème de Alc. fr. 10B voigT et Sapph. fr. 102 (voir p. ex. Nisbet \& Rudd 2004 ad loc).

64. Voir encore 3.19.25-27, où Télèphe, que cherche à séduire Rhodé dite tempestiua, est comparé à Vesper, l'astre du mariage ; l'Ode 3.20 n'a plus rien de nuptial, mais décrit le conflit d'un homme et d'une femme pour un bel éphèbe.

65. Pour une lecture de la séquence 3.26-3.28 comme un adieu progressif à l'amour et à la poésie avant les deux poèmes de clôture, voir Santirocco 1986 : 145-149.

66. Hor. carm. 3.27. 34-66 pour le monologue (o relictum / filiae nomen pietasque..., turpe comissum..., paelex), et 25-76 pour l'entier du récit, et en particulier, pour la révélation de Vénus, 73 : uxor inuicti Iouis esse nescis.

67. Lowrie 1997 : 297-316, en particulier 313-316.

68. Hor. carm.3.27.1-24; on rapprochera en particulier les v.13-14: sis licet felix, ubicumque

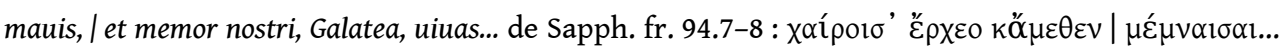
(voir Lowrie 1997 : 315).

\section{RÉSUMÉS}

Partant du constat que la réception de Sappho oppose les volets erotique et nuptial de sa poésie, cet article aborde le cas particulier de la lyrique d'Horace, qui ne semble pas faire de place à la Sappho nuptiale. Le thème du mariage y apparaît néanmoins de diverses manières. Il constitue d'abord un idéal moral, et ce surtout dans des odes politiques soutenant les réformes entreprises par Auguste et culminant dans les lois de 18-17 av. J.-C. sur les mariages et les adultères. Par contraste, les odes érotiques concernent des relations essentiellement non-matrimoniales, conformément à la tradition lyrique dominante. Néanmoins, un certain nombre de formules et d'images y introduisent des connotations nuptiales, qui prennent dans ce contexte des valeurs métaphoriques. Le mariage peut ainsi représenter la saison de la vie propice à l'amour ; moment unique dans une vie amoureuse et récurrent comme circonstance lyrique rituelle, il confronte alors le temps linéaire de l'amour à celui, cyclique, de la poésie lyrique. Mais le mariage fonctionne aussi comme métaphore de l'éternité non pas érotique, mais poétique promise par la lyrique, qui permet à Horace d'opposer sa poésie d'amour à celle de Catulle et des élégiaques. Ainsi, bien que la Sappho nuptiale ne soit pas directement évoquée par Horace - sans doute parce que Catulle l'avait fait avant lui -, le thème du mariage n'en occupe pas moins une place importante, entre autres dans la définition de sa lyrique par rapport à la tradition et aux poètes contemporains.

\section{INDEX}

Mots-clés : réception de Sappho, poésie d'amour, mariage, Horace, Catulle, lyrique, élégie 
AUTEUR

OLIVIER THÉVENAZ

Université de Lausanne

olivier.thevenaz@unil.ch 